Pontifícia Universidade $C_{\text {atólica }}$

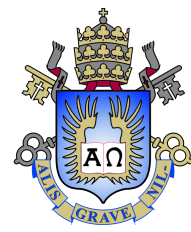

Guilherme Pereira Freire Machado

\title{
A Scenario Approach for Chance-constrained Short-term Scheduling with Affine Rules
}

Dissertation presented to the Programa de Pós-graduação em Engenharia Elétrica of PUC-Rio in partial fulfillment of the requirements for the degree of Mestre em Engenharia Elétrica.

Advisor: Prof. Álvaro de Lima Veiga Filho 

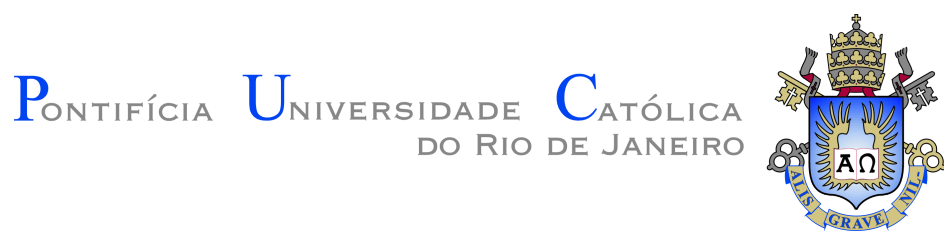

Guilherme Pereira Freire Machado

\section{A Scenario Approach for Chance-constrained Short-term Scheduling with Affine Rules}

Dissertation presented to the Programa de Pós-graduação em Engenharia Elétrica of PUC-Rio in partial fulfillment of the requirements for the degree of Mestre em Engenharia Elétrica. Approved by the Examination Committee:

Prof. Álvaro de Lima Veiga Filho

Advisor

Departamento de Engenharia Elétrica - PUC-Rio

Prof. Alexandre Street de Aguiar Departamento de Engenharia Elétrica - PUC-Rio

Prof. Davi Michel Valladão

Departamento de Engenharia Industrial - PUC-Rio

Prof. Bruno Fanzeres dos Santos Departamento de Engenharia Industrial - PUC-Rio 
All rights reserved.

\section{Guilherme Pereira Freire Machado}

Graduated in electrical engineering from the Federal University of Rio de Janeiro with a double degree in automated systems from the École Supérieure d'Électricité.

Bibliographic data

Machado, Guilherme Pereira Freire

A scenario approach for chance-constrained short-term scheduling with affine rules / Guilherme Pereira Freire Machado ; advisor: Alvaro Veiga. - 2020.

72 f.: il. color. ; $30 \mathrm{~cm}$

Dissertação (mestrado) - Pontifícia Universidade Católica do Rio de Janeiro, Departamento de Engenharia Elétrica, 2020.

Inclui bibliografia

1. Engenharia Elétrica - Teses. 2. Otimização estocástica. 3. Planejamento da operação. 4. Restrições probabilísticas. 5. Regras de decisão lineares. I. Veiga, Alvaro. II. Pontifícia Universidade Católica do Rio de Janeiro. Departamento de Engenharia Elétrica. III. Título.

CDD: 621.3 
To my parents, brothers, family and friends for support and encouragement. 


\section{Acknowledgments}

To my advisor Professor Alvaro Veiga for the encouragement, understanding and partnership during this work.

To PSR and PUC-Rio, for providing the conditions, without which this work could not have been carried out. For the discussions and the fertile environment for exchanging ideas.

To my parents, for the education, attention, affection and support that are essential at all hours.

To my colleagues at PSR and PUC-Rio, who shared this ambitious journey and made it much more enjoyable.

To the professors, for whom I have great admiration, and who were kind to participate in the examining committee.

To all professors and employees of the Department for the teachings and the help offered.

This study was financed in part by the Coordenação de Aperfeiçoamento de Pessoal de Nível Superior - Brasil (CAPES) - Finance Code 001. 


\section{Abstract}

Pereira Freire Machado, Guilherme; Veiga, Alvaro (Advisor). A Scenario Approach for Chance-constrained Short-term Scheduling with Affine Rules. Rio de Janeiro, 2020. 72p. Dissertação de Mestrado - Departamento de Engenharia Elétrica, Pontifícia Universidade Católica do Rio de Janeiro.

Multi-stage stochastic hydrothermal planning stands as one of the most critical problems in the power systems industry, mostly due to its vast implication in the system operation. The multi-stage stochastic hydrothermal scheduling refers to determining the economic dispatch of the power plants that minimize the global operation cost under the system's physical constraints. One of the main difficulties of the problem lies in the representations of uncertainty, as the dispatch decision must consider the different possible scenarios of water inflow, renewable generation, and the demand.

More recently, we have seen a worldwide speed up in the integration of variable renewable sources. Nonetheless, these sources have a greater uncertainty in the short-term than the world has ever experienced. Therefore, to support the dispatch scheduling, the models must accurately represent the uncertainties without increasing computational time.

In this work it is proposed a novel formulation for a multistage stochastic week-ahead economic dispatch with unit-commitment. The model uses affine decision rules to be computationally tractable. The relationship between the decision rules and the scenario approach is explored, and by building the uncertainty set with the scenario approach, both the feasibility of the decision rule policy and the chance-constraint on the load balance are respected.

\section{Keywords}

Stochastic optimization; Energy planning; Scenario-approach; Chanceconstraints; Affine rules. 


\section{Resumo}

Pereira Freire Machado, Guilherme; Veiga, Alvaro. Planejamento da operação no curto prazo com restrições probabilísticas e regras de decisão lineares usando uma abordagem com cenários. Rio de Janeiro, 2020. 72p. Dissertação de Mestrado

- Departamento de Engenharia Elétrica, Pontifícia Universidade Católica do Rio de Janeiro.

O planejamento hidrotérmico estocástico multi-etapa se destaca como um dos problemas mais importantes do setor elétrico, principalmente devido à sua grande relevância na operação do sistema. Este problema refere-se a determinar o despacho ótimo das usinas que minimizam o custo de operação sob as restrições físicas do sistema. Uma das principais dificuldades do problema reside nas representações de incerteza, pois a decisão de despacho deve considerar os diferentes cenários possíveis de afluência de água, geração renovável e demanda.

Mais recentemente, o grande aumento de fontes renováveis variáveis trouxe a atenção dos pesquisadores para como melhorar a granularidade do modelo sem aumentar muito o tempo computacional.

Neste trabalho é proposto uma nova formulação para um despacho econômico estocástico multi-etapa com unit-commitment. O modelo usa regras de decisão afins para ser computacionalmente tratável. A relação entre regras de decisão e o scenario approach é explorada e, ao construir o conjunto de incertezas, tanto a viabilidade da política da regra de decisão quanto a restrição probabilística do balanço de carga são automaticamente respeitadas.

\section{Palavras-chave}

Otimização estocástica; Planejamento da operação; Restrições probabilísticas; Regras de decisão lineares. 


\section{Table of contents}

$\begin{array}{llr}1 & \text { Introduction } & \mathbf{1 1}\end{array}$

$\begin{array}{lll}1.1 & \text { Objective } & 13\end{array}$

$\begin{array}{lll}1.2 & \text { Contributions } & 13\end{array}$

$\begin{array}{lll}1.3 & \text { Organization } & 13\end{array}$

2 Decision rules $\quad \mathbf{1 5}$

$\begin{array}{lll}2.1 & \text { Decision rule theory } & 15\end{array}$

2.2 Affine decision rules applied to hydrothermal scheduling 17

3 Scenario Approach 19

$\begin{array}{lll}3.1 & \text { Literature Review } & 19\end{array}$

3.2 Scenario Approach Theory 20

3.3 On Helly's dimension 22

4 Methodology 25

$\begin{array}{ll}\text { Nomenclature } & 25\end{array}$

$\begin{array}{lll}4.1 & \text { Introduction } & 28\end{array}$

4.2 Uncertainty 30

4.3 Multistage Stochastic Chance-Constrained Unit Commitment Model 33

$\begin{array}{lll}4.4 & \text { Solution Methodology } & 39\end{array}$

5 Discussion and further improvements 42

5.1 Discussion $\quad 42$

5.2 Electrical Areas 43

5.3 Affine policy per scenario cluster 44

5.4 Stochastic reserve requirement 44

6 Case Study $\quad 46$

6.1 Chilean system 46

6.2 Evaluation procedure 49

$\begin{array}{ll}6.3 \text { Model evaluation } & 51\end{array}$

6.4 Reserve requirement performance $\quad 56$

6.5 Improvements impact on the model 58

$\begin{array}{lll}6.6 & \text { Seasonality effect } & 60\end{array}$

$\begin{array}{lll}7 & \text { Conclusion } & 65\end{array}$

$\begin{array}{ll}\text { Bibliography } & 67\end{array}$ 


\section{List of figures}

Figure 3.1 Support constraint example. Image taken from [1] 23

Figure 6.1 Chilean system $\quad 46$

Figure 6.2 Installed capacity by technology 47

Figure 6.3 Total generation by technology in a typical week 48

Figure 6.4 SIC system uncertainty bounds 48

Figure 6.5 SING system uncertainty bounds 49

Figure 6.6 Two-phase procedure scheme 49

Figure 6.7 Planning procedure $\quad 50$

Figure 6.8 Reference scenario and uncertainty region 50

Figure 6.9 Total renewable production by scenario 51

Figure 6.10 Hydro generation $\quad 52$

Figure 6.11 Thermal generation $\quad 52$

Figure 6.12 Thermal flexibility and scheduled generation for T1-DET and T1-2STAGE 53

Figure 6.13 Thermal flexibility and scheduled generation for T1MSTAGE and T1-MSTAGE-CPP 54

Figure 6.14 Total true-up expected cost $\quad 54$

Figure 6.15 Total tru-up expected saving 55

Figure 6.16 Reserve requirement $\quad 56$

Figure 6.17 Thermal flexibility of T2-DET-RES 57

Figure 6.18 Total true-up expected cost 57

Figure 6.19 Total tru-up expected saving 58

Figure 6.20 Thermal flexibility of T3-MSTAGE-CLUS 59

Figure 6.21 Thermal flexibility of T3-MSTAGE-1AREA 59

Figure 6.22 Total true-up expected cost 60

Figure 6.23 Total true-up expected saving 60

Figure 6.24 Net demand's Root-mean-square deviation per month 61

Figure 6.25 Thermal flexibility and scheduled generation for T4-DET and T4-2STAGE 62

Figure 6.26 Thermal flexibility and scheduled generation for T4MSTAGE and T4-MSTAGE-CCP 63

Figure 6.27 Total true-up expected cost $\quad 63$

Figure 6.28 Total true-up expected saving 64 


\section{List of tables}

Table 6.1 Installed capacity by electrical area 47

Table 6.2 Description of tests cases employed to evaluate the benefit of the chance-constrained model 51

Table 6.3 Loss of load probability 53

Table 6.4 Description of features in the model T2-DET-RES 56

Table 6.5 Description of models T3-MSTAGE-1AREA and T3MSTAGE-CLUS 58

Table 6.6 Description of tests cases employed to evaluate the seasonality impact 61

$\begin{array}{lll}\text { Table } 6.7 & \text { Loss of load probability } & 62\end{array}$ 


\section{1 \\ Introduction}

Multistage stochastic hydrothermal planning is one of the most critical problems in the power systems industry, mostly due to its huge impact on electrical systems' operation. The multistage stochastic hydrothermal planning refers to determining the power plants' optimal dispatch that minimizes the global operation cost under the system's physical constraints. The main difficulty of the problem lies in both the unit-commitment constraints and on the representation of uncertainty.

The increase of variable renewable sources has recently turned the researcher's attention to improving the modeling of these uncertainties without increasing the computational time. Since uncertainties related to renewable sources have a granularity of hours, the multistage stochastic hydrothermal problem's difficulty has greatly increased as it needs to have at least hourly resolution. Ignoring these uncertainties is not an option since it could incur an unacceptable risk that the computed production schedule be significantly more costly. On the other side, incorporating uncertainty is challenging, especially due to the number of states and random variables.

There are several approaches regarding how to deal with uncertainty, each one with different practical and computational requirements [2]:

- Stochastic optimization - SO

- Robust optimization - RO

- Chance-constrained optimization - CCP

In stochastic optimization, each uncertainty is modeled as a random variable with a probability distribution. The usual solution method employed is using scenario trees, where the tree branches in each stage. However, scenario trees may cause a "state explosion" problem depending on the number of stages since the number of scenarios varies exponentially with the number of states.

The robust optimization approach has a better computational performance and does not need a probability distribution for the random variable; instead, it must have an uncertainty set. It is known that robust problems may give conservative solutions; therefore, it is necessary to apply techniques to restrict the uncertainty set with some "uncertainty budget" not to be too conservative. 
The chance-constrained approach considers a probability of violating the constraints involving the uncertainties, enforcing the feasibility of the constraints up to some desired level of reliability. In other words, it allows some violation of the constraints under a desired probability level, and in this sense, it could be seen as a relaxation of the robust problem. Unfortunately, even with the relaxation CCP turn out to be extremely difficult to solve [3].

The decision of which approach to take has a great impact and depends on the availability of data, the uncertainty type, and the time budget.

This work proposes a novel formulation for the hydrothermal week-ahead multistage stochastic chance-constrained dispatch. The model uses a future cost function as an input to approximate the water value of the hydro reservoirs. The problem has hourly resolution, where each hour is a stage. The uncertainties in the problem are the inflow $\iota_{i}$ and the net demand.

The hydrothermal planning problem is complex because it needs to schedule the operation in advance, without full knowledge of the future and with some plants with physical time-coupling constraints with considerable duration. For instance, some thermal plants may have weekly commitment and minimum up and downtime of more than days. Furthermore, some hydro plants may have considerable travel times of days or weeks. Therefore, the week ahead planning problem has an adequate time length to capture most of the system's slow dynamic.

A stochastic model with hourly resolution permits a detailed analysis of the system operation instead of usual approximations used in long-term models, such as load-duration curves. Stochastic optimization is essential to correctly capture the impacts of variable renewable sources in the system and the dynamic of decision under uncertainty.

Nonetheless, solving a week-ahead multistage stochastic problem with hourly duration (168 stages) is a challenging goal. Multistage stochastic models are usually solved with scenario tree methods [4], which unfortunately suffer from the "curse of dimensionality": the tree needs to branch whenever a new uncertainty is revealed. Therefore the number of scenarios grows exponentially with the number of decision stages.

This methodology could be used as a support tool for the short-term operation in a rolling horizon scheme, where the model is run each day with a one-week horizon; however, only the next-day decision is implemented.

The state variables of the model are reservoir storage and thermal plant commitment decisions. The reservoir storage is evaluated with the future cost function in the last hour of the week. The future cost function is a piece-wise linear function and is an input for the problem. 


\section{1}

\section{Objective}

This work aims to solve a multistage stochastic programming problem with chance-constraints and unit-commitment, where each hour is one stage and the problem has a one-week duration. As presented before, this is a very difficult task, and in order to accomplish it, some compromise was made for the sake of tractability.

In this work, concepts of $\mathrm{SO}, \mathrm{RO}$, and $\mathrm{CCP}$ were used to derive the final formulation with the chance-constrained problem. It is proposed a novel affine formulation to solve the multistage stochastic problem within the computational requirements. The chance-constraints solution methodology is based on the scenario approach $[3,5]$, in a framework developed in [6].

This work presents a novel tractable formulation for a multistage stochastic problem with chance-constraints based on the scenario approach theory's latest developments. Furthermore, the idea of using the scenario approach to ensure the feasibility of the affine policy in out-of-sample scenarios with probabilistic properties is also explored.

\section{2}

\section{Contributions}

This Thesis's contribution are the following:

1. A new tractable formulation for a multistage stochastic unit-commitment with chance-constraints

2. A data-driven model with no assumption over the probability distribution, provided that enough samples are available

3. A method to create probabilistic feasibility cuts for the decision rules, which guarantees that they are feasible with any desired probability

4. A new method to create a reserve requirement from any stochastic solution

\section{3}

\section{Organization}

The remainder of this work is organized as follows. Chapter 2 presents the theory of decision rules and their relation to stochastic problems. Chapter 3 reviews the main theorems and concepts around the scenario approach theory. Chapter 4 develops the methodology and formulates the multistage stochastic problem with chance-constraints. Chapter (5) presents a discussion about the 
decision rule, an improvement to use clusters of decision rules, and a novel reserve requirement methodology. A case study with the Chilean system is presented in Chapter 6. Finally a conclusion is drawn in Chapter 7. 


\section{2}

\section{Decision rules}

\section{1}

\section{Decision rule theory}

A decision rule is a vector-valued function that maps a random variable into a decision $\delta: \Xi \rightarrow \mathcal{D}$, where $\mathcal{D}$ is the $n$-dimensional decision space and $\Xi$ is the uncertainty space [7]. This approach is built under the assumption of hazard-decision, i.e., once the uncertainty is revealed, the decision rule maps the uncertainty to the recourse decision. Therefore, the decision rule applies to second-stage decisions.

The second stage value function of a multistage optimization problem is:

$$
\begin{aligned}
Q(x, \xi)= & \min _{g} q^{T} \cdot g \\
& \text { s.t. } \\
& T(\xi) \cdot x+W(\xi) \cdot g=h(\xi) \\
& g \in \mathcal{G}
\end{aligned}
$$

The decision rule approach is based on the idea that the second stage optimal decision can be mapped into a functional form $\delta$, such as:

$$
\begin{gathered}
\mathcal{D}=\mathcal{G} \\
\delta^{*}(\xi)=g^{*}(\xi)
\end{gathered}
$$

Where:

$$
g^{*}(\xi)=\underset{g}{\operatorname{argmin}}\left\{q^{T} \cdot g \mid T(\xi) \cdot x+W(\xi) \cdot y=h(\xi), g \in \mathcal{G}\right\}
$$

When (2-3) holds true, we say the decision rule is optimal. The decision rule modifies the stochastic second-stage problem to a search of the optimal decision rule, instead of the original problem that searches for the value of $g$ 
that minimizes $q^{T} \cdot g$ over the feasible space. It is noteworthy that when in possession of such a decision rule, the decision output is mapped for no matter the uncertainty. Therefore, the problem is no more one that minimizes $g$ under the known uncertainty $\xi$, but is the one of finding $\delta$, i.e., the decision rule function:

$$
\begin{aligned}
& \min _{\delta} \mathbb{E}\left[q^{T} \cdot \delta(\xi)\right] \\
& \text { s.t. } \\
& T(\xi) \cdot x+W(\xi) \cdot \delta(\xi)=h(\xi), \quad \xi \in \Xi \\
& \delta(\xi) \in \mathcal{D}
\end{aligned}
$$

Remark 2.1 The decision rule problem is an uncertain optimization problem.

So, with the decision rule approach, a two-stage problem assumes the form of:

$$
\begin{aligned}
& \min _{x, \delta} c^{T} \cdot x+\mathbb{E}\left[q^{T} \cdot \delta(\xi)\right] \\
& \text { s.t. } \\
& A \cdot x=b \quad, x \in \mathbb{R}_{+}^{n} \\
& T(\xi) \cdot x+W(\xi) \cdot \delta(\xi)=h(\xi), \quad \xi \in \Xi \\
& \delta(\xi) \in \mathcal{D}
\end{aligned}
$$

Sometimes, approximations are made to simplify the problem (2-6), and the functional form of $\delta$ is limited to a class of vector valued mapping $\mathcal{D} \subset \mathcal{G}$, which is a subset of the original domain, since to obtain an analytical description of $\delta^{*}$ might be difficult if not impossible [7].

Amongst the functional class of decision rules, the simplest one is the constant policy. The second simplest one is the affine policy, where the decision rule is an affine function of the uncertainty. The functional form options to use as a decision rule are enormous, but the most explored in the literature is the affine function. Extensions of the affine formulation for stochastic optimization are developed in [8], where it also highlights that affine decision rules may lead to infeasible instances even for stochastic problems with complete recourse. Other more generic decision rules are presented in [9] and [10].

In the work of [7], it was proved that for a solvable two-stage stochastic program with concave recourse, there exists a function in the class of piecewise 
affine functions which determines an optimal decision rule for the second stage.

Although affine decision rules may cause a loss of optimality [11] for other problems, in [12] the authors also cited the potential of complexity reduction of affine decision rules on multistage stochastic programming. The quality of the affine decision rule approximation may depend on the problem structure.

Furthermore, [11] compares affine decision rules with a Sample Average Approximation (SAA) for a stochastic inventory problem. The authors conclude that affine decision rules scale well in the number of stages considered. In contrast, the computational time for the SAA variants for more than four to seven stages explodes.

The reason for the better performance of the affine decision rules compared to the SAA is that it allows to employ a robust technique over the uncertainty set, which has a tremendous computational gain.

The importance of affine decision rules was recently revived in the context of robust optimization after [13] used it to build a tractable approximation for a multistage decision process. After that, [14] used affine decision rules to solve a multistage portfolio optimization problem.

A multistage adaptive robust optimization for the unit-commitment problem was presented in [15], where the uncertainty is in the nodal net loads. Multistage optimization could be formulated as a deterministic equivalent problem with the use of affine policy. This could be done because affine policy automatically respects the nonanticipativity constraint. In the results, the model proposed by [15] outperformed the deterministic unit-commitment, as well as existing two-stage robust unit-commitment models.

\section{2}

\section{Affine decision rules applied to hydrothermal scheduling}

The literature has several applications of affine policy in power systems operation. [16] and [17], proposed to use affine policies for operating electrical reserves in power systems. To exemplify, the model used in [16] is illustrated in Equation (2-7) where $u_{i}$ is the power injection input of generator $i, e_{i}$ is the nominal schedule, $D_{i}$ is the linear coefficient and $\varepsilon$ is the forecast error.

In [16] and [17], the authors used the affine linear coefficients $\left(D_{i}\right)$ to determine the participation factor of each generator in the reserve operation. It reported cost reduction by using such a policy in comparison with other reserve operations. Afterward, the formulation was extended, and the affine policy idea was applied to a multistage unit-commitment problem by [18]. 


$$
u_{i}=D_{i} \varepsilon+e_{i}
$$

Affine decision rules were also explored in the optimal power flow field. In [19], was employed an adjustable robust optimal power flow model with affine policy. In the paper, the author also explored the affine coefficients, but in this case, to use as participation factors for the automatic generation control. Another affine decision rules application of optimal power flow was developed in [20], where it also used an affinely adjustable robust optimization approach, but in this case, to calculate the amount of reactive power of photovoltaic inverters in a distribution network.

In [21], affine decision rules are employed in a multistage hydropower scheduling model that maximizes profit. It considered two sources of uncertainty: energy prices and inflow. The model was validated for four hydro plants in Norway. The paper shows that the model is promising concerning the feasibility of hydropower operations and that the complexity reduction, due to affine decision rules, led to short computational times. 


\section{Scenario Approach}

In this chapter, the scenario approach theory is introduced. A literature review of the main finding of the scenario approach is presented in Section (3.1), giving an overview of this recent field's major advances. Later, in Section (3.2), the scenario approach is gently introduced, and the main aspects of the theory are highlighted.

This chapter aims to present the main aspects of the scenario approach; therefore, proofs are not presented. For a more formal presentation, the reader should refer to $[22,5,1,23]$.

\section{1}

\section{Literature Review}

Chance-constrained programming problems have a long history, being introduced by Charnes, Cooper, and Symonds [24] and with extensive contributions since. The main challenge of chance-constrained programming problems lies in the fact that the chance-constraints are very hard to compute and that the feasible set is often non-convex [25].

Recent approaches that emerged to solve chance-constrained problems are the employment of sample average approximation [25], where Pagnoncelli et al. replaces the chance-constraint by an empirical probability measure with binary variables, the use of convex approximations of chance-constraints [26], and to discretize the probability distribution and solve the combinatorial problem [27].

The scenario approach [3] is another recent approach to solve chanceconstrained programming problems and was applied in this work due to considerable advantages against the previous. The scenario approach advantages are: it does not need any assumption over the probability distribution of the uncertainty, it does not need to add new binary variables, and it is generic for any convex chance-constraint.

The scenario approach theory was first introduced in the seminal paper [3], as an alternative to solve uncertain convex problems. In [3], Calafiori et al. showed that by solving a Scenario Approach Program, i.e., a problem with sampled constraints from the uncertainty set, the solution only violates a small portion of the out-of-sample constraints, under Assumptions (3.2) and (3.3). 
In [28], the authors applied the scenario approach to a robust control design problem and provided tighter bounds to the sample size. Furthermore, they provided a bound for the probability that the result's violation probability is greater than what was previously defined: $\mathbb{P}\left\{\mathbb{V}\left(g^{*}\right)>\epsilon\right\}$.

The bound on $\mathbb{P}\left\{\mathbb{V}\left(g^{*}\right)>\epsilon\right\}$ is further refined in [29], where Campi et al. proved that it is bounded by a binomial tail, for uncertain convex problems that always admits an optimal solution.

Calafiori developed a new theory for the scenario approach, which he called Random Convex Programs [22]. He arrived at the same results as [29], but in a different manner where the assumption on feasibility is not needed. Also, in [22], Calafiori discussed the idea of random convex programs with violated constraints and showed the relation between uncertain problems and chance-constrained problems. Furthermore, an upper bound for the optimal objective value of the chance-constrained problem related to the random convex problem is proved. At the same time, Campi et al. developed similar results in [5] but with different assumptions.

Schildbach et al. extended the scenario approach theory to multistage decision problems [1]. In this class of problems, a single (or multiple) chanceconstraint(s) involves only a subset of the decision variables, i.e., the chanceconstraint(s) affect only a certain subspace of the decision space. Schildbach et al. [1] argues that the size of the scenarios must be related to the "support dimension" and not to the "problem dimension", thus decreasing the number of scenarios needed with the same probabilistic guarantees. The paper proved the validity of the scenario approach theory's theorems to the concept of "support dimension".

Finally, Zhang et al. [30] provided an extensive study of support dimension and provided a different bound based on the uncertainty space. Both Zhang's and Schildbacks' bounds are valid, and one does not dominate the other, so ultimately, both bounds have to be checked in order to verify the tightest.

\section{2}

\section{Scenario Approach Theory}

The scenario approach gives an approximation to a chance-constrained problem. This approximation does not need any assumption on the random variable distribution, and it could be data-driven. However, the number of samples needs to respect the Inequality (3-3) to guarantee the feasibility and optimality with the chosen reliability level.

The scenario approach is a technique to solve problems in the form of Program (3.1). Where, $\mathbf{g} \in \mathbb{R}^{i}$ is the decision vector, $\mathbf{c} \in \mathbb{R}^{i}$ is the cost vector, 
and $\mathcal{G} \subset \mathbb{R}^{i}$ is the compact and convex domain of $\mathbf{g}$. $\varepsilon$ denotes all uncertainty quantities, whose sample space $\Xi$ is of generic nature. The constraint (3-1b) is a chance-constraint, containing a constraint function $\mathbf{L}: \mathbb{R}^{i} \times \Xi \rightarrow \mathbb{R}$, of both the decision variable and the uncertainty, and $1-\epsilon$ is the probability level of the chance-constraint.

Definition 3.1 (uncertain convex program) The following program is an uncertain convex program:

$$
\begin{array}{ll}
\min _{\mathbf{g}} & \mathbf{c}^{T} \cdot \mathbf{g} \\
\text { s.t. } & \mathbb{P}[\mathbf{L}(\mathbf{g} ; \varepsilon) \geq 0] \geq 1-\epsilon \\
& \mathbf{g} \in \mathcal{G}
\end{array}
$$

It is worth mentioning that although a linear function represents the objective function, the scenario approach accepts any convex function. The linear function was chosen only for the sake of simplicity.

The following assumptions about the Program (3.1) are required to apply the scenario approach:

Assumption 3.2 (convexity) The Program (3.1) is convex and the constraint function $\mathbf{L}(\cdot ; \varepsilon)$ is convex for almost every uncertainty $\varepsilon \in \Xi$.

Assumption 3.3 (uncertainty) There exist a probability measure $\mathbb{P}$ on $\Xi$, which may be unknown, but there is a sufficient number of independent random samples available.

\subsection{1}

\section{Scenario Approach Program}

The scenario approach provides a computationally tractable approximation to Program (3.1):

Definition 3.4 (scenario approach program) Let $\Xi_{N}$ be a set of $N$ i.i.d samples of $\Xi$. Under Assumptions (3.2) and (3.3), the scenario approach program is:

$$
\begin{array}{ll}
\min _{\mathbf{g}} & \mathbf{c}^{T} \cdot \mathbf{g} \\
\text { s.t. } & \mathbf{L}(\mathbf{g} ; \varepsilon) \geq 0 \quad \forall \varepsilon \in \Xi_{N} \\
& \mathbf{g} \in \mathcal{G}
\end{array}
$$


The scenario approach program replaces the chance-constraint (3-1b) by (3-2b), which is a deterministic constraint over the i.i.d samples $\Xi_{N}=$ $\left\{\varepsilon^{(1)}, \varepsilon^{(2)}, \varepsilon^{(3)}, \ldots, \varepsilon^{(N)}\right\}$.

\section{2 .2}

\section{Sample Size}

In order for the scenario approach program (3.4) be an approximation of the uncertain convex program (3.1), the number of samples must obey the following inequality [29]:

$$
N \geq \frac{2}{\epsilon}\left(\ln \frac{1}{\beta}+\zeta-1\right)
$$

Where, $\epsilon \in(0,1)$ is the chance-constraint's risk parameter, $\beta \in(0,1)$ is the sample confidence parameter, and $\zeta$ is the program's Helly's dimension as defined in (3.5). If the number of scenarios $N$ satisfy (3-3), then with probability greater or equal to $1-\beta$, it holds that the solution of the scenario approach program (3.4) is feasible in the uncertain convex program (3.1).

Definition 3.5 (Helly's dimension) The Helly's dimension of a scenario approach program is the smallest integer $\zeta$ that upper bounds the essential supremum [31] of the program's number of support constraints.

The support constraints are defined next, and Figure (3.2.2) illustrates an optimization problem's support constraints.

Definition 3.6 (support constraint) A constraint of an optimization problem is a support constraint if its removal entails a change in the objective function.

\section{3}

\section{On Helly's dimension}

Helly's dimension plays a central role in the scenario approach, as the sample size grows linearly with it. If the problem is fully-supported, [29] proved that Helly's dimension is equal to the dimension of the decision space. Nonetheless, for general problems, computing Helly's dimension is often challenging [30]. Therefore the approach employed by the literature is to find valid upper bounds. The first upper bound was proved by [22], where Calafiori found that the decision space dimension was a valid upper bound for any problem.

The definition of a fully-supported problem is given below: 


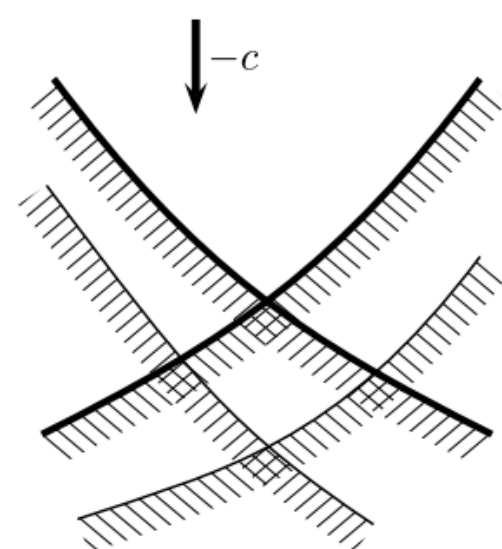

(a) Two Support Constraints.

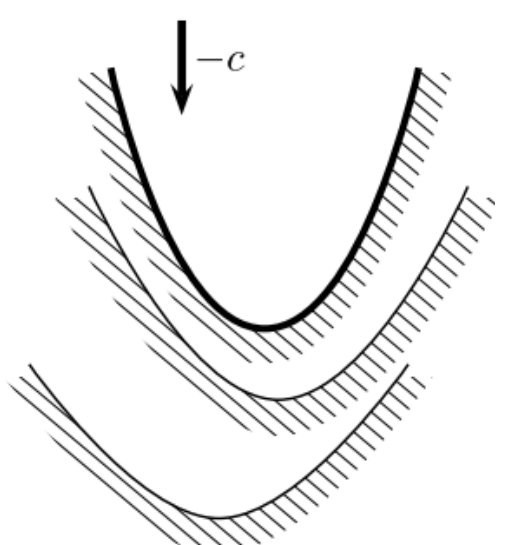

(b) One Support Constraint.

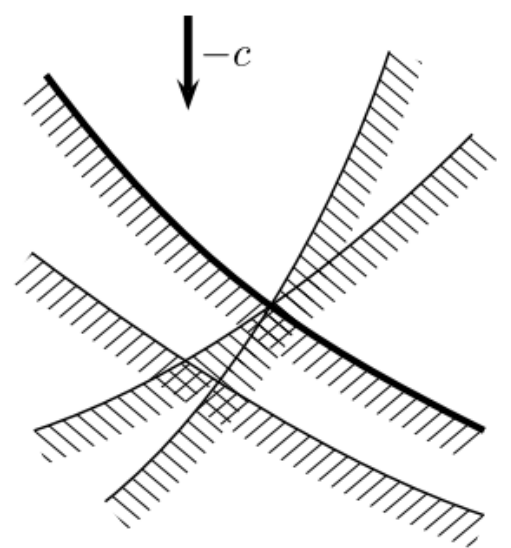

(c) One Support Constraint.

Figure 3.1: Support constraint example. Image taken from [1]

Definition 3.7 (fully-supported problem) The scenario approach program is fully-supported if its number of support constraints is $d$ with probability 1. Where $d$ is the dimension of the decision space.

Moreover, the number of fully-supported problems is indeed reduced. Therefore, finding better bounds on Helly's dimension allows for a faster solution to the problem and also reducing the conservationism.

Shildbach et al. [1] investigated the application of the scenario approach in multistage problems. A first remark about multistage problems is they are often not fully supported. A second remark is that they often have a special structure, where the uncertain constraint does not involve all the decision variables simultaneously. One of the advances proposed by Shildbach et al. was to extend the scenario approach theory for the cases where the chance-constraint involves only a subset of the decision variables.

Shildbach et al. proposed to explore the structure of the uncertain constraint and its dimension, which they called "support dimension". The paper's main result is that the uncertain constraint with a lower support dimension than the problem's dimension needs a lower number of samples. 
The support dimension has a similar definition to Helly's dimension, differing only that the support dimension is restricted to a chance-constraint:

Definition 3.8 (support dimension) The support dimension of a scenario approach program is the smallest integer $\zeta_{i}$ that upper bounds the essential supremum [31] of support constraints of a chance-constraint $i$.

Furthermore, Shildbach et al. provided an upper bound for the support dimension in case the value is not known exactly. The support dimension is bounded by the "support rank":

Definition 3.9 (support rank) The support rank is the dimension $d$ of the decision space less the maximal dimension of an (almost surely) unconstrained subspace. The unconstrained subspace is a linear subspace of $\mathbb{R}^{d}$ that cannot be constrained by the sampled instances of the chance-constraint for almost every value of the sample $\Xi_{N}$.

Another series of works that significantly developed the theory on Helly's dimension is Zhang et al. [23, 30]. In [23], the authors bounded Helly's dimension by the number of active constraints for the case where constraints are affine over the uncertainty. Following, in [30], the authors provided a systematic study of Helly's dimension bounds exploiting the structure of the constraint functions with respect to the uncertainty. The bounds derived in [30] does not dominates nor is dominated by the one derived in [1].

In [30], Zhang et al. provide an upper bound for Helly's dimension for the case where the chance-constraint does not offer enough structure for the support rank to improve the bounds but has some special structure regarding the uncertainty. 


\section{Methodology}

\section{Nomenclature}

This section provides a description of the notation. Bold symbols are reserved to matrix (upper-case) and vectors (lower-case). Sets are written in calligraphic font $(\mathcal{C})$. The hat is used for forecasted scenario $\hat{\varepsilon}$ or the decision variables in the forecasted scenario $\hat{u}$

\subsection{1}

\section{Sets}

$\mathcal{H}$ Set of hours.

$\mathcal{I}$ Set of hydro plants.

$\mathcal{I}(a)$ Set of hydro plants of area $a$.

$\mathcal{J}$ Set of thermal plants.

$\mathcal{J}(a)$ Set of thermal plants of area $a$.

$\mathcal{L}$ Set of Interconnections.

$\mathcal{F}(a)$ Set of interconnections that are connected to area $a$, with positive sense leaving area.

$\mathcal{T}(a)$ Set of interconnections that are connected to area $a$, with positive sense entering area.

$\mathcal{M}(i)$ Set of hours.

$\mathcal{A}$ Set of electrical areas.

$\Xi$ Original uncertainty set.

$\Xi_{N}$ Uncertainty set with $\mathrm{N}$ samples from $\Xi$.

$\mathcal{U}$ Uncertainty set obtained with scenario approach.

$\mathcal{S}$ Uncertainty set obtained by sampling $\Xi_{N}$.

$\mathcal{H}$ Set of hours.

$\mathcal{B}^{*}$ Hyper-rectangle built from the solution of Margellos's intermediary problem. 


\subsection{2}

\section{Indexes}

$h$ Hours.

$s$ Scenarios.

$i$ Hydro plants.

$m$ Hydro plants immediately upstream of another plant $i$.

j Thermal plants.

a Electrical area.

$l$ Interconnection.

$a(j)$ Area of thermal plant $j$.

$a(i)$ Area of thermal plant $i$.

\subsection{3}

\section{Constants}

$\varepsilon_{h, a}$ Net demand at hour $h$, area $a$ and scenario.

$c_{j}$ Thermal plants operational cost.

$\iota_{i}$ Incremental inflow to hydro plant $i$ at scenario.

$R_{j}^{u p}$ Maximum ramp up rate of thermal plant $j$.

$R_{j}^{d n}$ Maximum ramp down rate of thermal plant $j$.

$U P_{j}$ Minimum up-time of thermal plant $j$.

$D T_{j}$ Minimum down-time of thermal plant $j$.

$\rho_{i}$ Production factor of hydro plant $i$.

$\bar{g}_{j}$ Maximum generation of thermal plant $j$.

$v_{i}^{0}$ Initial storage of hydro plant $i$.

$\bar{v}_{i}$ Maximum storage at hydro plant $i$.

$\underline{v}_{i}$ Minimum storage at hydro plant $i$.

$\bar{u}_{i}$ Maximum turbined outflow of hydro plant $i$.

$\underline{u}_{i}$ Minimum turbined outflow of hydro plant $i$.

$\bar{f}_{i}$ Maximum power flow of line $l$.

$\underline{f}_{i}$ Minimum power flow of line $l$.

$N$ Number of samples.

$\epsilon$ Chance-constraint risk level.

$\beta$ Scenario approach confidence level. 
$\zeta$ Helly's dimension of optimization problem.

$H$ Number of hours in the problem (Cardinality of set $\mathcal{H}$ ).

$A$ Number of electrical areas in the problem (Cardinality of set $\mathcal{A}$ ).

$I$ Number of hydro plants in the problem (Cardinality of set $\mathcal{I}$ ).

$J$ Number of thermal plants in the problem (Cardinality of set $\mathcal{J}$ ).

$S$ Number of scenarios in the stochastic problem (Cardinality of set $\mathcal{S}$ ).

\subsection{4}

\section{Functions}

$\alpha_{i}$ Future cost function of hydro plant $i$.

$\mathbf{L}(\varepsilon)$ Net load balance matrix.

$L_{h, a}(\varepsilon)$ Net load balance equation for hour $h$ and electrical area $a$.

$\mathbf{W}\left(\varepsilon_{h}\right)$ Water balance matrix.

$W_{h, i}\left(\varepsilon_{h}\right)$ Water balance equation for hour $h$ and hydro plant $i$.

$p(\varepsilon)$ Probability of realization $\varepsilon$.

\subsection{5}

\section{Decision variables}

$g_{h, j}$ Generation of thermal plant $j$ at hour $h$.

$x_{h, j}$ Commitment of thermal plant $j$ at hour $h$.

$y_{h, j}$ Binary variable indicating if thermal plant $j$ was started at hour $h$.

$z_{h, j}$ Binary variable indicating if thermal plant $j$ was shut-down at hour $h$.

$v_{i}$ Stored volume of hydro plant $i$ at final hour.

$u_{h, i}$ Turbined outflow of hydro plant $i$ at hour $h$.

$w_{h, i}$ Spillage of hydro plant $i$ at hour $h$.

$f_{i}$ Power flow through line $l$.

$\lambda_{h, i}$ Linear coefficient of affine decision rule for hour $h$ and hydro plant $i$.

$\omega_{h, j}$ Linear coefficient of affine decision rule for hour $h$ and thermal plant $j$. 


\section{1 \\ Introduction}

System operators use the unit-commitment problem to create a commitment schedule for the generation units in the following days or weeks. This problem must consider the uncertainty and guarantee that the schedule is feasible, respecting the electrical system's physical constraints.

The renewable sources' variability and uncertainty increase the difficulty of operating the electrical systems. Nowadays, new types of resources, such as ramping and flexibility capabilities, are necessary to be scheduled in order to maintain the system's reliable operation due to the variability and uncertainty of renewable sources.

Stochastic and probabilistic methods are recommended for power systems' planning, as they can help maintain and improve the system reliability despite the increasing penetration of renewable sources [32].

The uncertainty in dispatch scheduling is of two types [33]: forecast error and unreliable equipment. Forecast errors are often continuous, such as inflow and demand forecast. On the other hand, equipment failure is mostly discrete, such as generation and line outages.

Most recently, the uncertainty related to forecast errors started gaining more relevance since, with the high penetration of variable renewable sources, the underlying uncertainty of the system has increased [34].

Since ignoring such a substantial increase in uncertainty level may occur unacceptable risks [35], it became necessary to model the uncertainty in largescale optimization problems. Nonetheless, there is still a gap to improve existing models, especially when it comes to chance-constrains.

We proposed a new multistage stochastic model with chance-constraints over the loss of load probability (LOLP) in this work. To the best of the authors' knowledge, the only other model representing both a stochastic operation and chance-constraints is [36], where the authors model a two-stage stochastic problem with chance-constraints, to guarantee with high probability that the amount of wind shedding is low. Nonetheless, their approach is significantly different from ours since they solve a two-stage unit-commitment. They combine sample-average approximation (SAA) with the chance-constraints replaced by equivalent deterministic linear inequalities.

Depending on the electrical system's size, the deterministic unit commitment problem may already be challenging to solve [32]. Consequently, considering uncertainty in the unit-commitment problem poses a significant challenge [37], especially when the multistage dynamic is considered. 
Additionally, chance-constraints increase the problem's difficulty in several degrees, as it is non-convex and known to be NP-Hard [38, 39].

Until now, the literature still does not have a final solution for multistage stochastic problems [34]. However, the literature offers different approaches to solve it, each with its advantages and disadvantages.

Stochastic unit-commitment usually utilize scenario-based techniques to represent the uncertainty in the unit-commitment formulation. There are two classes of stochastic problems: two-stage and multistage. In two-stage models, the uncertainty is treated statically. It is revealed only once, and the decisions are split into the first stage (before information is revealed) and the second stage (after information is revealed). In the first stage, the commitment is decided, and in the second stage, the generation is scheduled, knowing the uncertainty realization fully.

Multistage models treat the uncertainty dynamically, and decisions are made as information is revealed. The decisions are constantly being adjusted as there is more information available. The benefit of using multistage models is that the interaction between decision-making and uncertainty unfolding is better represented, making the model more realistic and significantly reducing the expected operation cost [32]. However, multistage unit commitment is significantly harder to solve than the two-stage models due to the increased number of stages that suffers from the "curse of dimensionality".

A major line of research in stochastic multistage unit commitment is developing efficient decomposition algorithms [40, 41, 42, 43, 44, 45]. However, these algorithms cannot still address large real-life problems, as they are usually applicable only to a relatively small scenario tree and may have scalability issues with practical problems [37].

Most recently, Zou et al. [37] proposed to apply the Stochastic Dual Dynamic Programming (SDDP) [46] approach to solve multistage unit-commitment problems, with the variant known as SDDiP [47]. This approach can deal with larger scenario trees; nonetheless, it demands stage-wise independence.

This work follows another line, aiming to simplify the original problem to obtain the solution in a tractable time. It follows the same reasoning presented by Shapiro et al.[12] where the authors argue that it is acceptable to make a compromise and simplify the problem to solve it in a reasonable time. Additionally, Shapiro et al.[12] proposed to use linear decision rules to exploit the uncertainty set structure, therefore conferring tractability to a multistage stochastic problem.

We propose a linear decision rule model that exploits the structure of the uncertainty set. This model uses the forecast prediction as a reference 
scenario, where we model the unit-commitment without simplifications. In other scenarios, we apply a simplification on the decision variables, and an affine decision rule substitutes the decision variables.

The downside of this approach is that the affine decision rule restrain the decision's feasible space; therefore, it possibly leads to sub-optimal solutions. Nonetheless, it is a compromise in order to be able to represent the uncertainty better.

\subsection{1}

\section{Chapter Structure}

This chapter structure is the following: in Section (4.2), the uncertainty model and the chance-constraint are presented. The multistage stochastic formulation with chance-constraints is presented in Section (4.3) and the solution methodology is presented in Section (4.4).

\section{2}

\section{Uncertainty}

In this work, we chose to model the renewable output and demand uncertainties through the net demand variable $\varepsilon \in \mathbb{R}^{H \times A}$. The net demand is the hourly sum of all the load minus all the renewable generation connected to an electrical area, where the electrical area is an abstraction that represents one region in the network topology. The electrical area can be one bus or a set of buses. We chose to model the uncertainties aggregated spatially in the electrical area to decrease the number of random variables, decreasing the number of necessary scenarios and computational burden, as will be discussed in the following sections.

We represent an uncertain constraint (4-1) related to the load balance of each electrical area, where the net demand is uncertain and belongs to the uncertainty set $\Xi$.

$$
\begin{array}{r}
\sum_{j \in J(a)} g_{h, j}+\sum_{i \in I(a)} \rho_{i} \cdot u_{h, i}+\sum_{l \in F(a)} f_{h, l} \\
-\sum_{l \in T(a)} f_{h, l}=\varepsilon_{h, a} \quad \forall a, h, \varepsilon \in \Xi
\end{array}
$$

$g_{h, j}$ is the thermal generation of thermal plant $j$ on hour $h ; u_{h, i}$ is the turbining of hydro plant $i$ on hour $h ; f_{h, l}$ is the power flow in the interconnection $l$ on hour $h, \varepsilon_{h, a}$ is the net demand of area $a$ in hour $h$, and $\rho_{i}$ is the production factor of hydro plant $i . I(a), J(a)$ are respectively the set of hydro and thermal 
plants inside the electrical area $a ; F(a), T(a)$ are the set of interconnections leaving and entering the electrical area $a$.

For the sake of simplifying the notation, consider the net load balance matrix $\mathbf{L}(\varepsilon) \in \mathbb{R}^{H \times A}$, for the index $(h, a)$ :

$$
\begin{aligned}
\mathbf{L}(\varepsilon)_{h, a}= & \sum_{j \in J(a)} g_{h, j}+\sum_{i \in I(a)} \rho_{i} \cdot u_{h, i}+\sum_{l \in F(a)} f_{h, l} \\
& -\sum_{l \in T(a)} f_{h, l}-\varepsilon_{h, a}
\end{aligned}
$$

The joint-chance constraint of the loss of load probability (LOLP) related to the uncertainty is:

$$
\mathbb{P}[\mathbf{L}(\varepsilon) \geq 0] \geq 1-\epsilon
$$

\subsection{1}

\section{Scenario Approach}

The scenario approach is a methodology to solve uncertain convex optimization problems. This methodology uses a set of $N$ i.i.d samples $\Xi_{N}:=\left\{\varepsilon^{(n)}\right\}_{n=1}^{N}$ from the uncertainty set $\Xi$, to solve a scenario problem, where the uncertain constraint is substituted by a set of deterministic constraints that must be feasible in $\Xi_{N}$.

The scenario approach can be used to approximate chance-constrained problems [5], where the chance-constraint (4-3) is substituted by the scenario approach constraints (4-4).

$$
\mathbf{L}(\varepsilon) \geq 0 \quad \forall \varepsilon \in \Xi_{N}
$$

The validity of the equivalence between (4-3) and (4-4) is guaranteed by the sampled set's $\Xi_{N}$ size. The sampled problem has an apriori defined violation probability $(\epsilon)$ and an associated validity probability $(1-\beta)$ that dictates the minimum number of necessary samples N [29]. Provided that the relationship (4-5) between violation, validity, and samples are respected, it is proven that the approximation is valid [22].

$$
N \geq \frac{2}{\epsilon} \cdot\left(\zeta-1+\ln \frac{1}{\beta}\right)
$$


Where, $N$ is the number of scenarios, $\beta \in(0,1)$ is the risk of failure, or the solution's confidence, $\epsilon \in(0,1)$ is the constraint's violation probability, and $\zeta$ is the Helly's dimension [22], which is upper bounded by the number of decision variables in the problem.

Nonetheless, the downside of the scenario approach is still the number of samples, which may turn the problem computationally intractable. Since $N$ practically scales in $\mathcal{O}\left(\frac{\zeta}{\epsilon}\right)[30]$, a vast attention in the literature were turned on finding tighter bounds for $\zeta[1,23,30]$, when the problem is not fully-supported $[22]$.

Modaressi et al. [48] solved a look-ahead real-time economic dispatch in a 2000-bus synthetic grid using the scenario approach. However, the scenario approach demands the convexity of the uncertain problem, so it is not directly applied for unit-commitment problems [49].

\subsection{2}

\section{Uncertainty Set With Scenario Approach}

Most recently, two papers were published that use the scenario approach to solve power system's problems [50, 49]. In [50], the authors solve an N-1 secure day-ahead dispatch with chance-constraints in an IEEE 30 bus system, and in [49], the authors propose a framework for the uncertain unit-commitment using the scenario approach. In this work, we apply the same methodology as in $[50,49]$ to solve the chance-constraints, i.e., based on building an uncertainty set using the scenario approach and solving a robust counterpart as proposed in $[6]$.

The advantage of using the scenario approach instead of using other approaches $[51,52,53,54,55,56]$ is that this is a purely data-driven approach, i.e., no assumption over the uncertainty distribution is made, and this approach provides probabilistic guarantees over the representation of the uncertainty set.

Margellos et al. [6] suggests splitting the uncertain problem in two, where first an intermediary scenario approach problem is used to obtain the bounds of the uncertainties. Then a robust counterpart of the uncertain problem is employed with the obtained bounded uncertainty set. The benefits of this approach are two-fold, first it avoids entering a high number of constraints in the scenario approach problem, which may already be computationally intensive, and secondly, it relaxes the condition on the convexity of the uncertain problem since the scenario approach is applied to an intermediary problem, only this intermediary must be convex.

The intermediary problem (4-6) is a hyperrectangle minimization problem, where it's goal is to find probabilistic bounds for the uncertainty using the 
scenario approach theory. These bounds will provide an uncertainty set that carries the probability used, considering the number of samples defined by $(4-5)$.

$$
\begin{array}{cl}
\min _{\bar{\tau}, \underline{\tau} \in \mathbb{R}^{H \times A}} & \sum_{a \in A} \sum_{h \in H} \bar{\tau}_{h, a}-\underline{\tau}_{h, a} \\
\text { s.t. } & \mathbb{P}[\varepsilon \in \Xi \mid \varepsilon \in[\bar{\tau}, \underline{\tau}]] \geq 1-\epsilon
\end{array}
$$

Where, $\bar{\tau}_{h, a}, \underline{\tau}_{h, a}$ are respectively the upper and lower bound of the net demand uncertainty at hour $h$ and electrical area $a$; and $\Xi$ is the net demand original uncertainty set.

Note the scenario approach version of problem (4-6) still has a high number of constraints, nonetheless the simple structure of the problem allows us to solve it using any linear optimization solver.

Then, the scenario approach uncertainty set $\mathcal{U}$ is built from the hyperrectangle $\mathcal{B}^{*}:=\times_{a=1}^{A} \times_{h=1}^{H}\left[\bar{\tau}_{h, a}^{*}, \underline{\tau}_{h, a}^{*}\right]$ that is built from the solutions $\bar{\tau}^{*}, \underline{\tau}^{*}$ of the intermediary problem (4-6).

$$
\begin{aligned}
& \mathcal{U}:=\mathcal{B}^{*} \cap \Xi \\
& \mathcal{U}:=\left\{b \in \Xi \mid b_{h, a} \in\left[\bar{\tau}_{h, a}^{*}, \underline{\tau}_{h, a}^{*}\right] \forall a \in \mathcal{A}, h \in \mathcal{H}\right\}
\end{aligned}
$$

Margellos et al. [6], states that when $\mathcal{U}$ is applied in the robust counterpart of the uncertain problem, there is a confidence of at least $1-\beta$, that the solution respects the original chance-constraint. The constraint (4-8) shows the robust version of the chance-constraint in the robust counterpart problem.

$$
\min _{h \in \mathcal{H}, a \in \mathcal{A}} \min _{\varepsilon \in \mathcal{U}} \mathbf{L}_{h, a}(\varepsilon) \geq 0
$$

\section{3}

\section{Multistage Stochastic Chance-Constrained Unit Commitment Model}

\subsection{1}

\section{Deterministic Unit Commitment}

The deterministic unit commitment formulation is as follows: 


$$
\begin{aligned}
& \min \sum_{h \in \mathcal{H}} \sum_{j \in \mathcal{J}} c_{j} \cdot g_{h, j}+c_{j}^{s p} \cdot y_{h, j}+c_{j}^{d n} \cdot z_{h, j}+\sum_{i \in \mathcal{I}} \alpha_{i}\left(v_{i}\right) \\
& \text { s.t. } v_{i}=v_{i}^{0}+\iota_{i}+\sum_{h \in \mathcal{H}}\left[-w_{h, i}-u_{h, i}\right. \\
& \left.+\sum_{m \in M(i)}\left(w_{h, m}+u_{h, m}\right)\right] \quad \forall i \\
& \underline{v}_{i} \leq v_{i} \leq \bar{v}_{i} \quad \forall i \\
& \underline{u}_{i} \leq u_{h, i} \leq \bar{u}_{i} \quad \forall i \\
& \underline{g}_{j} x_{h, j} \leq g_{h, j} \leq \bar{g}_{j} x_{h, j} \quad \forall j \\
& x_{h, j}, y_{h, j}, z_{h, j} \in\{0,1\} \quad \forall j, h \\
& x_{h, j}-x_{h-1, j}=y_{h, j}-z_{h, j} \quad \forall j, h \geq 2 \\
& t+U T_{j}-1 \\
& \sum_{t=h} x_{t, j} \geq U T_{j} \cdot y_{t, j} \\
& \forall j, h \in\left\{1, \ldots, H-U T_{j}+1\right\} \\
& \sum_{t=h}^{H}\left(x_{t, j}-y_{t, j}\right) \geq 0 \\
& \forall j, h \in\left\{H-U T_{j}+1, \ldots, H\right\} \\
& \sum_{t=h}^{t+D T_{j}-1}\left(1-x_{t, j}\right) \geq D T_{j} \cdot z_{t, j} \\
& \forall j, h \in\left\{1, \ldots, H-D T_{j}+1\right\} \\
& \sum_{t=h}^{H}\left(1-x_{t, j}-z_{t, j}\right) \geq 0 \\
& \forall j, h \in\left\{H-D T_{j}+2, \ldots, H\right\} \\
& g_{h+1, j}-g_{h, j} \leq R_{j}^{u p} \quad \forall j \\
& g_{h+1, j}-g_{h, j} \geq-R_{j}^{d n} \quad \forall j \\
& \sum_{j \in J(a)} g_{h, j}+\sum_{i \in I(a)} \rho_{i} \cdot u_{h, i}+\sum_{l \in F(a)} f_{h, l} \\
& -\sum_{l \in T(a)} f_{h, l}=\hat{\varepsilon}_{h, a(i)} \quad \forall a, h \\
& \underline{f_{l}} \leq f_{h, l} \leq \bar{f}_{l} \quad \forall h, l
\end{aligned}
$$

Where $c_{j}$ is the operative cost for thermal plant $j ; v_{i}^{0}$ is the initial storage of hydro plant $i ; \iota_{i}, \rho_{i}$ are respectively the inflow and production factor of hydro plant $i ; R_{j}^{u p}, R_{j}^{d n}$ are respectively the ramp up and down of thermal plant $j$; $D T_{j}, U T_{j}$ are respectively the minimum down-time and up-time. $\alpha_{i}$ is the cost to go function of hydro plant $i$ and $a(i), a(j)$ are respectively the electrical area 
of hydro plant $i$ and thermal plant $j . g_{h, j}$ is the thermal generation of thermal plant $j$ on hour $h ; v_{i}$ is the final storage of hydro plant $i ; w_{h, m}$ and $u_{h, i}$ are respectively the spillage and turbining of hydro plant $i$ on hour $h ; x_{h, j}, y_{h, j}, z_{h, j}$ are respectively the commitment status, start-up and shut-down variables of thermal plant $j$ on hour $h$; and $f_{h, l}$ is the power flow in the interconnection $l$ on hour $h . \mathcal{H}$ is the set of hours; $\mathcal{I}$ is the set of hydro plants; $\mathcal{J}$ is the set of thermal plants; $\mathcal{L}$ is the set of interconnections; $\mathcal{A}$ is the set of electrical areas; $I(a), J(a)$ are respectively the set of hydro and thermal plants inside the electrical area $a ; F(a), T(a)$ are the set of interconnections leaving and entering the electrical area $a$; $h$ is the index of hours; $i$ is the index of hydro plants; $j$ is the index of thermal plants; $l$ is the index of interconnections; $a$ is the index of electrical areas.

The future cost function is considered an input to this model, and it could be created through an SDDP [57] execution, for instance.

Equation (4-9b) describes hydro plants' water mass balance; Constraints (4-9c) and (4-9d) model the hydro plants' reservoir and turbining limits; Constraint (4-9e) define the thermal plants' operational limits; Constraints (4-9f)-(4-9k) are the unit commitment formulation[58]; Equations (4-9l) and (4-9m) model the thermal plants' ramps; Equation (4-9n) enforce the power balance; and Constraint (4-9o) define the power flow limits.

\subsection{2}

\section{Multistage Stochastic Unit Commitment}

In multistage stochastic models, the decision-making process is dynamic. There is a sequence of decisions, followed by the reveal of the uncertainty and another decision, progressively until the end of the simulation horizon as it is represented in (4-10).

$$
x_{1} \rightarrow \varepsilon_{1} \rightarrow x_{2} \rightarrow \ldots
$$

The decisions made after the reveal of information are called "recourse decisions". For this specific type of decision, an alternative method called decision rule can be applied, where the decision variable is substituted by a function of the uncertainty [7]. A decision rule is a vector-valued function that maps a random variable into a decision $\delta: \Xi \rightarrow \mathcal{D}$, where $\mathcal{D}$ is the $n$-dimensional decision space and $\Xi$ is the uncertainty space [7].

In this model, we apply affine decision rules in both the hydro and thermal 
generation as follows:

$$
\begin{array}{r}
u_{h, i}(\varepsilon)=\hat{u}_{h, i}+\lambda_{h, i} \cdot\left(\varepsilon_{h, a(i)}-\hat{\varepsilon}_{h, a(i)}\right), \\
\forall i \in \mathcal{I}, h \in \mathcal{H} \\
g_{h, j}(\varepsilon)=\hat{g}_{h, j}+\omega_{h, j} \cdot\left(\varepsilon_{h, a(j)}-\hat{\varepsilon}_{h, a(j)}\right), \\
\forall j \in \mathcal{J}, h \in \mathcal{H}
\end{array}
$$

The decision rules are a function of the electrical area's $a$ uncertain net demand, $\varepsilon_{h, a}$, in hour $h$ and the functions $a(i)$ and $a(j)$ are used to map the hydro and thermal plant index to the electrical area it belongs. The decision rules can be interpreted as a linear deviation from the generation $\hat{u}_{h, i}$ and $\hat{g}_{h, j}$ in the forecasted scenario $\hat{\varepsilon}$, similar to $[17,18]$.

The parametrization of the affine policy is carefully chosen to be the net demand $\varepsilon_{h, a}$, since the model can profit from two advantages: the load balance equation (4-12a) over the uncertainty set can be substituted by equations (4-12b) and (4-12c); also, the load balance equation is respected for any out-of-sample scenario if the affine decision rule is applied.

$$
\begin{array}{rlr}
\sum_{j \in J(a)} g_{h, j} & +\sum_{i \in I(a)} \rho_{i} \cdot u_{h, i} & \\
& +\sum_{l \in F(a)} \hat{f}_{h, l}-\sum_{l \in T(a)} \hat{f}_{h, l}=\varepsilon_{h, a} & \\
\Uparrow & \forall a, h, \varepsilon \in \Xi \\
\sum_{i \in I(a)} \rho_{i} \cdot \lambda_{h, i}+\sum_{j \in J(a)} \omega_{h, j}=1 & \\
\sum_{j \in J(a)} \hat{g}_{h, j}+\sum_{i \in I(a)} \rho_{i} \cdot \hat{u}_{h, i} & \forall a, h \\
+\sum_{l \in F(a)} \hat{f}_{h, l}-\sum_{l \in T(a)} \hat{f}_{h, l}=\hat{\varepsilon}_{h, a} &
\end{array}
$$

The multistage stochastic unit-commitment formulation with the linear decision rules is displayed in (4-13).

$$
\min \sum_{h \in \mathcal{H}} \sum_{j \in \mathcal{J}} c_{j}^{s p} \cdot y_{h, j}+c_{j}^{d n} \cdot z_{h, j}+\mathbb{E}\left[\sum_{h \in \mathcal{H}} \sum_{j \in \mathcal{J}} c_{j} \cdot g_{h, j}\left(\varepsilon_{h}\right)+\sum_{i \in \mathcal{I}} \alpha_{i}\left(v_{i}\left(\varepsilon_{h}\right)\right)\right]
$$

s.t. Constraints $(4-9 f)-(4-9 k)$ for $(x, y, z)$

Constraints $(4-11 a),(4-11 b),(4-12 b)$ and $(4-12 c)$ 


$$
\begin{aligned}
& W_{h, i}\left(\varepsilon_{h}\right)=0 \\
& \underline{v}_{i} \leq v_{i}\left(\varepsilon_{h}\right) \leq \bar{v}_{i} \\
& \underline{u}_{i} \leq u_{h, i}\left(\varepsilon_{h}\right) \leq \bar{u}_{i} \\
& \underline{g}_{j} x_{h, j} \leq g_{h, j}\left(\varepsilon_{h}\right) \leq \bar{g}_{j} x_{h, j} \\
& g_{h+1, j}\left(\varepsilon_{h}\right)-g_{h, j}\left(\varepsilon_{h}\right) \leq R_{j}^{u p} \\
& g_{h+1, j}\left(\varepsilon_{h}\right)-g_{h, j}\left(\varepsilon_{h}\right) \geq-R_{j}^{d n} \\
& \underline{f l}_{l} \leq \hat{f}_{h, l} \leq \bar{f}_{l}
\end{aligned}
$$$$
\forall i, h, \varepsilon \in \Xi
$$$$
\forall i, \varepsilon \in \Xi
$$$$
\forall i, \varepsilon \in \Xi
$$

$\forall j, \varepsilon \in \Xi$

$\forall j, \varepsilon \in \Xi$

$\forall j, \varepsilon \in \Xi$

In order to improve readability, we substituted the water balance (4-9b) equation for the set of functions $W_{h, i}(\cdot)$ defined in (4-14):

$$
\begin{array}{r}
W_{h, i}\left(\varepsilon_{h}\right):=v_{i}\left(\varepsilon_{h}\right)-v_{i}^{0}-\iota_{i}-\sum_{h \in \mathcal{H}}\left[-w_{h, i}\left(\varepsilon_{h}\right)\right. \\
\left.-u_{h, i}\left(\varepsilon_{h}\right)+\sum_{m \in M(i)}\left(w_{h, m}\left(\varepsilon_{h}\right)+u_{h, m}\left(\varepsilon_{h}\right)\right)\right]
\end{array}
$$

In (4-13) $\mathbb{E}[\cdot]$ denotes the expectation with respect to the random parameter $\varepsilon$, and constraints (4-15b)-(4-15h) are ensured $\mathbb{P}$-a.s.. Since we apply linear decision rules on the decision variables, the non-anticipativity of the dynamic decision is already satisfied, and we can omit the conditional expectation in the constraints $\mathbb{E}\left[\cdot \mid \varepsilon^{h}\right][59]$.

\subsection{3}

\section{Multistage Stochastic Chance-Constrained Unit Commitment}

The multistage stochastic chance-constrained unit-commitment formulation (4-15) is obtained by adding the chance-constraint over the LOLP (4-3) to the problem (4-13). 


$$
\min \sum_{h \in \mathcal{H}} \sum_{j \in \mathcal{J}} c_{j}^{s p} \cdot y_{h, j}+c_{j}^{d n} \cdot z_{h, j}+\mathbb{E}\left[\sum_{h \in \mathcal{H}} \sum_{j \in \mathcal{J}} c_{j} \cdot g_{h, j}\left(\varepsilon_{h}\right)+\sum_{i \in \mathcal{I}} \alpha_{i}\left(v_{i}\left(\varepsilon_{h}\right)\right)\right]
$$

s.t. Constraints $(4-9 f)-(4-9 k)$ for $(x, y, z)$

Constraints $(4-11 a),(4-11 b),(4-12 b)$ and $(4-12 c)$

Constraint $(4-3)$

$W_{h, i}\left(\varepsilon_{h}\right)=0$

$\forall i, h, \varepsilon \in \Xi$

$\underline{v}_{i} \leq v_{i}\left(\varepsilon_{h}\right) \leq \bar{v}_{i}$

$\forall i, \varepsilon \in \Xi$

$\underline{u}_{i} \leq u_{h, i}\left(\varepsilon_{h}\right) \leq \bar{u}_{i}$

$\forall i, \varepsilon \in \Xi$

$$
\underline{g}_{j} x_{h, j} \leq g_{h, j}\left(\varepsilon_{h}\right) \leq \bar{g}_{j} x_{h, j}
$$

$\forall j, \varepsilon \in \Xi$

$$
\begin{aligned}
& g_{h+1, j}\left(\varepsilon_{h}\right)-g_{h, j}\left(\varepsilon_{h}\right) \leq R_{j}^{u p} \\
& g_{h+1, j}\left(\varepsilon_{h}\right)-g_{h, j}\left(\varepsilon_{h}\right) \geq-R_{j}^{d n} \\
& \underline{f_{l}} \leq \hat{f}_{h, l} \leq \bar{f}_{l}
\end{aligned}
$$$$
\forall j, \varepsilon \in \Xi
$$$$
\forall j, \varepsilon \in \Xi
$$

It is worth noting that in the formulation (4-3), the joint-chance constraint only involves the generation, ensuring a specified reliability on the LOLP. Nonetheless, no probability is ensured at the transmission line overload probability (TLOP)[60]. Therefore the fundamental assumption in order for the validity of the chance-constraint is that inside each electrical area $a$, there is no transmission constraint.

The multistage stochastic chance-constrained unit-commitment formulation provides a solution that profits from both modeling methods: it ensures a reliability level through the LOLP and at the same time solves the problem for a least expected cost operation. Models such as this that joins these two methodologies are not common in the literature due to each one's intrinsic difficulty separately.

Another application in the literature that joins stochastic and chanceconstraint approach is [36], where the authors want to guarantee with high probability that the amount of wind shedding is low. Nonetheless, their approach is significantly different than ours since they solve a two-stage unit-commitment, 
and they combine sample-average approximation (SAA) with the chanceconstraints replaced by equivalent deterministic linear inequalities.

\section{4}

\section{Solution Methodology}

This work leverages from the scenario approach to model the chanceconstraint and affine decision rules to model a multistage stochastic policy, which minimizes the expected cost.

The chance-constraints are solved using the method described at (4.2.2), where the chance-constraint is replaced for a robustified constraint over the uncertainty set $\mathcal{U}$.

The affine decision rules are applied in a set of discrete scenarios $\mathcal{S}$ obtained by sampling the uncertainty set $\Xi_{N}$. The reason for that is that the set $\Xi_{N}$ is proven to contain, with high probability, the range of minimum and maximum possible realizations; nonetheless this uncertainty set have a cardinality too big to be considered in this multistage stochastic unit-commitment problem. The alternative proposed is to sample it for a lower number of scenarios that give a tractable problem.

$$
\mathcal{S} \subset \Xi_{N}
$$

We notice that the fact that we are sampling the uncertainty set $\Xi_{N}$ for the stochastic optimization does not invalidate the chance-constraints since they are completely independent. As discussed previously, the scenario approach uncertainty set is obtained prior to this stochastic problem, and for the scenario approach robust constraint, we do not modify the uncertainty set $\mathcal{U}$.

The complete model is displayed below:

$\min \sum_{h \in \mathcal{H}} \sum_{j \in \mathcal{J}} c_{j}^{s p} \cdot y_{h, j}+c_{j}^{d n} \cdot z_{h, j}+\sum_{\varepsilon \in \mathcal{S}} \sum_{h \in \mathcal{H}} \sum_{j \in \mathcal{J}} p(\varepsilon) \cdot\left[c_{j} \cdot g_{h, j}\left(\varepsilon_{h}\right)+\sum_{i \in \mathcal{I}} \alpha_{i}\left(v_{i}\left(\varepsilon_{h}\right)\right)\right]$

s.t. Constraints $(4-9 f)-(4-9 k)$ for $(x, y, z)$

$$
\text { Constraints }(4-11 a),(4-11 b),(4-12 b) \text { and }(4-12 c)
$$

$\mathbf{L}(\varepsilon)_{a, h} \geq 0 \quad \forall \varepsilon \in \mathcal{U}, a \in \mathcal{A}, h \in \mathcal{H}$

$W_{h, i}\left(\varepsilon_{h}\right)=0$ $\forall i, h, \varepsilon \in \mathcal{S}$ 


$$
\begin{array}{ll}
\underline{v}_{i} \leq v_{i}\left(\varepsilon_{h}\right) \leq \bar{v}_{i} & \forall i, \varepsilon \in \mathcal{S} \\
\underline{u}_{i} \leq u_{h, i}\left(\varepsilon_{h}\right) \leq \bar{u}_{i} & \forall i, \varepsilon \in \mathcal{S} \\
\underline{g}_{j} x_{h, j} \leq g_{h, j}\left(\varepsilon_{h}\right) \leq \bar{g}_{j} x_{h, j} & \forall j, \varepsilon \in \mathcal{S} \\
g_{h+1, j}\left(\varepsilon_{h}\right)-g_{h, j}\left(\varepsilon_{h}\right) \leq R_{j}^{u p} & \forall j, \varepsilon \in \mathcal{S} \\
g_{h+1, j}\left(\varepsilon_{h}\right)-g_{h, j}\left(\varepsilon_{h}\right) \geq-R_{j}^{d n} & \forall j, \varepsilon \in \mathcal{S} \\
\underline{f_{l} \leq \hat{f}_{h, l} \leq \bar{f}_{l}} & \forall h, l, \varepsilon \in \mathcal{S}
\end{array}
$$

Where, $p(\varepsilon)$ is the probability associated with the realization $\varepsilon$ and is defined as $p(\varepsilon):=\frac{1}{S}$.

\subsection{1}

\section{Feasibility cuts}

We propose to apply the bounds obtained at (4-7) to create feasibility cuts for the decision rule. These feasibility cuts ensure that the decision rule will have probabilistic guarantees to be feasible when applied in any out-of-sample scenario, with reliability $1-\beta$.

The feasibility cuts are developed analysing the structure of the constraints in (4-17) that have the decision rule. For the sake of simplicity, we will show the two possible cases in a generic decision rule $f(\varepsilon)=\lambda \cdot(\varepsilon-\hat{\varepsilon})+\hat{f}$. First, consider the simple case where we have a lower bound on the decision rule:

$$
\begin{gathered}
f(\varepsilon) \geq 0 \quad \forall \varepsilon \in \mathcal{U} \\
\mathbb{\sharp} \\
f\left(\underline{\tau}^{*}\right) \geq 0
\end{gathered}
$$

Then a second more complex case is when there is a box constraint, such as $(4-17 d)$. The box constraint can be substituted by separating the box constraint into two different inequalities and applying (4-19) to each one; and the case where we have temporal constraints, as in (4-17h), can be solved by taking the bounds that stress the constraint, such as the following: 


$$
g_{h+1, j}\left(\bar{\tau}_{h+1}^{*}\right)-g_{h, j}\left(\underline{\tau}_{h}^{*}\right) \leq R_{j}^{u p}
$$

Furthermore, we point out another advantage of the feasibility constraints: constraints $(4-17 \mathrm{~b})$ can be substituted by the feasibility constraints. This substitution will achieve the same reliability of the chance-constraints due to the equivalence obtained by our modeling approach, displayed by equations (4-12) where the modeling of the decision rule guarantees that in any realization, the load balance equality is valid. Consequently, we must only guarantee that the decision rule is feasible for the realizations with a probability $1-\epsilon$, which is accomplished by the feasibility cuts. 


\section{5 \\ Discussion and further improvements}

\section{1 \\ Discussion}

In the reference scenario, the generation variables are first stage decisions, while in the others they are affine functions of the net demand uncertainty. Both affine functions and decision variables respect the physical capacity of the respective plants.

The operation on the entire horizon of the reference scenario occurs in the first stage of the problem, since it is a coefficient in the affine rule that is the same for all second stage scenarios. Therefore, the forecast scenario is deterministic, i.e. the whole forecast is assumed known at the moment of the first stage decision.

The generation on the reference scenario $\hat{u}_{h, i}$ and $\hat{g}_{h, j}$ are used as the translation coefficient of the affine functions. As a consequence, this formulation has the same interpretation as $[16,17,18]$, where the uncertainty $\varepsilon$ is a prediction error that deviates from the forecast and the linear coefficient $\lambda, \omega$ determines how the decision variables will adapt with respect to the prediction error. The decision rule can also be interpreted as a feasible redispatch under the uncertainty set.

This formulation is similar to that of [15], where the authors proposed an multistage adaptative robust affine model for unit-commitment. Therefore, Proposition 3. of [15], which states that the load balance in all scenarios is resumed to two equations due to the affine decision rule also applies to this case. The difference between this approach and that of [15] is that we propose to solve a multistage stochastic problem instead of a robust problem and that in this model the translation coefficient also have a physical meaning, as generation in forecast of the operation. Furthermore, [15] presents a constraint generation algorithm that is proved to converge, since the uncertainty considered is a bounded polyhedron, which could also be used here.

The advantage of using affine decision rules is that it reduces the difficulty of the computationally hard multistage stochastic problem. The linear decision rule reduces the number of variables of the problem to be a linear function of 
the number of stages, furthermore the decision rules does not depends on the number of scenarios.

In the literature, the affine decision rules are often explored together with assumptions regarding the uncertainty set. [12] first proposed to use affine decision rules in stochastic problems, were the authors proposed to explore a polyhedral structure of the uncertainty set in order to make a multistage stochastic problem tractable. [11] compared a affine decision rule formulation, with a polyhedral uncertainty set, with the SAA method for different branching factors applied in an inventory problem. The authors showed that the affine decision rule had similar accuracy than the SAA with superior scalability, regarding the number of stages, presenting lower computational time. It is worth noting that this does not guarantee that the affine approximation has the same accuracy in the hydrothermal dispatch problem, it is rather used as an example of improvement due to the affine decision rule.

Finally, Bodur et al. [61] applied a different approach to multistage problems, where the affine decision rules are applied only to the state variables. This modelling allows a multistage problem to be treated as a two-stage problem, which drastically reduces the computational burden. Nonetheless, this approach is not possible in the hydrothermal dispatch problem, since this class of problems have time-coupling constraints that we do not have full control, such as the reservoir state variable, i.e. it is not possible to increase top of cascade reservoirs level when the decision rules need to.

\section{2 \\ Electrical Areas}

Network representation is famous for increasing the computational time. The optimal power flow problem, which represents the complete network equations, is known to be nonconvex and computationally hard. There are known models that are simpler than the optimal power flow, for instance, the DC model. Nonetheless, we chose to adopt a simplified model, since the main focus of this work is the stochastic problem.

In this work, the network was simplified with a transport model, i.e. representing only the interconnection limit, from each electrical area. This representation models the limit of the power exchange between the areas.

The author would like to highlight that the electrical area representation is not a network model, although it is modeled only the interconnection flows between areas, instead it is a key factor in the affine model, since the netload deviation in each electrical area is supplied only with the resources within that area, due to constraint (??). Another network model could also be adopted and 
it is suggested as a future work to investigate the impact of a more detailed network representation in the model.

\section{3}

\section{Affine policy per scenario cluster}

The affine policy stipulates that the same policy is applied to all scenarios, but in reality applying the same affine policy for scenarios with different characteristics (e.g. regular, extreme low and extreme high renewable generation) may be inefficient, since each cluster may need different types of resources and in different level. One alternative is to use a different policy for each cluster of scenarios, which allows for each cluster to have a better policy.

With one policy per scenario cluster, each cluster contains a set of scenarios grouped by similarity, using any available metric. For instance, this could result in different policies for high and low net demands, or even, dry and wet scenarios.

We describe a procedure for grouping these scenarios:

1. Calculate, for each net demand scenario $s$, the hourly deviations from the reference scenario - the deviation is measured in MW or GW.

2. For each scenario, calculate a scalar, $\Delta^{s}$, representing the magnitude of each scenario's deviations over time (e.g., this scalar could be the mean or a combination of mean and the CVaR of the deviations).

3. Group the scenarios according to the scalars $\Delta^{s}-$ if there is just one electrical area, the set of scalars is one-dimensional.

4. Solve the unit-commitment problem with one affine policy per cluster.

The affine coefficients now depend on the cluster $k$ :

$$
u_{h, i, k}\left(\varepsilon^{s}\right)=\hat{u}_{h, i, k}+\lambda_{h, i, k} \cdot\left(\varepsilon_{h, a}^{s}-\hat{\varepsilon}_{h, a}\right)
$$

For each scenario $\varepsilon^{s}$ belonging to the cluster $k$.

\section{4}

\section{Stochastic reserve requirement}

The necessity of a reserve requirement in an optimization model arises from the fact that some uncertainty is not being modeled explicitly in the problem or that a solution more conservative is adequate for the problem.

Nonetheless, in any stochastic formulation that explicitly represents the uncertainty, such as the formulation proposed in this work, it is not necessary to have an explicit reserve constraint [33] within the optimization model. 
In this section, it is proposed a methodology to extract a reserve requirement from an stochastic solution. Which, for the best of the author knowledge, is an original proposition.

The reserve requirement in power systems is the amount of additional power that a set of plants (synchronized or not) must be able to supply to the system in a stress scenario to maintain the balance between generation and system demand. In this work, it is proposed to calculate this additional amount by the difference in generation between the forecast scenario and the other scenarios considered in the simulation. This deviation quantifies what reserve margin would be needed if the Operator plans for the reference scenario, but instead another scenario is realized.

In other words, the proposed reserve requirement is a random variable:

$$
\begin{aligned}
& \tilde{b}_{h, j}=\tilde{g}_{h, j}-\hat{g}_{h, j} \\
& \quad \text { or } \\
& \tilde{b}_{h, i}=\rho_{i} \cdot \tilde{u}_{h, i}-\rho_{i} \cdot \hat{u}_{h, i}
\end{aligned}
$$

This procedure defines one random variable, $\tilde{b}_{h, i}$, for each hour. A risk measure $\gamma$ can be used to define an dynamic (hourly) deterministic reserve requirement, attending a desired risk aversion criteria.

$$
b_{h, i}=\gamma\left(\tilde{b}_{h, i}\right) \quad \forall h, i
$$

It is worth mentioning that the reserve requirement defined in this section is both dynamic and probabilistic, which guarantees that at every hour the reserve requirement has the same risk level.

For instance, a worst-case approach can be applied (as shown in the test cases) to the stochastic dispatch to build the reserve requirement. The robust criterion considers that the reserve requirement needed is equivalent to what the system would need to mitigate the effect of the in-sample variations of the net demand, seen in the planning.

The affine rules calculated by the stochastic model determine, for each hour and scenario, the amount of redispatch (i.e., the necessary corrective action) of each generator in relation to a reference scenario. The stochastic model also ensures that these redispatches are operationally viable: for example, if the capacity of generator A is $100 \mathrm{MW}$ and the reference generation at a given time is $90 \mathrm{MW}$, the maximum redispatch that could result from the affine rules for this generator, at this time, it would be $100-90=10 \mathrm{MW}$. 


\section{6}

\section{Case Study}

In this chapter, the performance of the proposed methodology is evaluated in the Chilean system. First, the Chilean system is described in Section (6.1), then the evaluation method is presented in Section (6.2), and the test results are presented in the Sections (6.3), (6.4), (6.5), and (6.6).

The Section (6.3) evaluates the benefit of the affine multistage chanceconstrained model, comparing it to different benchmarks. Section (6.4) evaluates the benefit of the proposed reserve requirement method, simulating a deterministic model with the reserve requirement and comparing its results with those obtained in the previous Section. Finally, Section (6.5) tests the impact of considering electrical areas and scenarios' clusters, and Section (6.6) evaluates the impact of the seasonality in the model.

\section{1}

\section{Chilean system}

The Chilean system is primarily thermal and has relevant hydroelectric and renewable (wind and solar) participation. An overview of the system is displayed in Figure 6.1.

The system has approximately $23 \mathrm{GW}$ of installed capacity. It comprises 189 thermal plants, 99 hydroelectric plants, and 97 renewable plants (solar and wind). Figure 6.2 shows the installed capacity percentage of each technology.
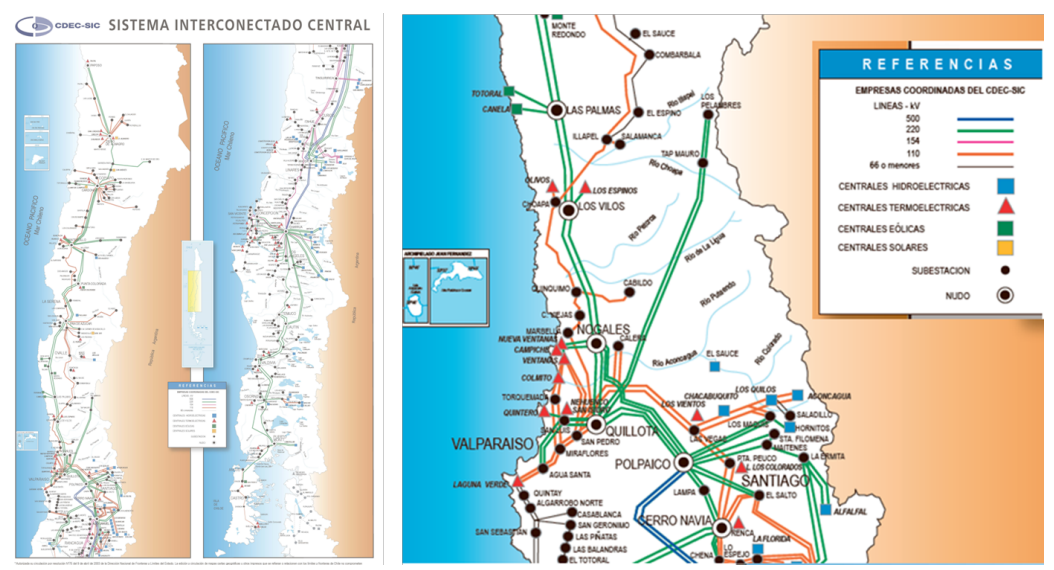

Figure 6.1: Chilean system 
Two electrical areas were modeled in the test cases: SIC (Central and Southern System) and SING (Northern system). We chose the areas by spatial separation. SING is an area mainly composed of mining load, with low variability along the day. SIC is where Santiago and the residential load are located. The network considered consists of a transport model between the two areas. The interconnection limit is $1500 \mathrm{MW}$. Table 6.1 shows the installed capacity of each technology for both SIC and SING.

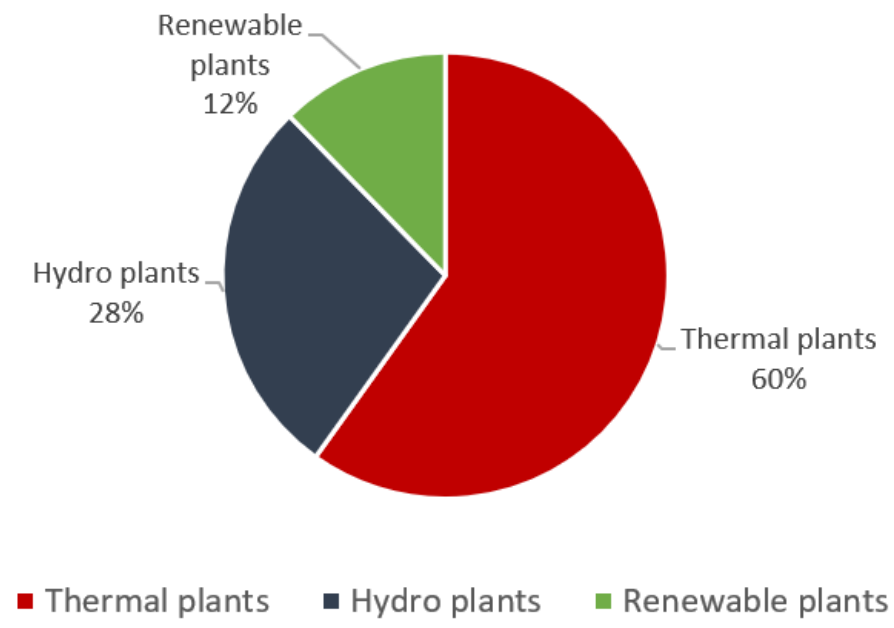

Figure 6.2: Installed capacity by technology

Table 6.1: Installed capacity by electrical area

\begin{tabular}{lccc}
\hline Technology & SIC $[\mathrm{MW}]$ & SING $[\mathrm{MW}]$ & Total $[\mathrm{MW}]$ \\
\hline Thermal & 8670.37 & 5101.12 & 13771.49 \\
Hydro & 6391.69 & 0 & 6391.69 \\
Renewable & 2096.9 & 736.3 & 2833.2 \\
\hline
\end{tabular}

Figure 6.3 shows the stacked generation by technology in a typical week. We can see that hydroelectric holds the most importance, but renewable and thermoelectric sources are also essential.

\subsection{1}

\section{Dataset}

In this case study, the Chilean dataset is of propriety of the consulting company PSR - Energy Consulting and Analytics. The dataset was built from the official Chilean data, with 54 scenarios of hourly renewable generation generated through reanalysis. The dataset contains all the existing hydro, thermal, and renewable plants available in the Chilean system. 


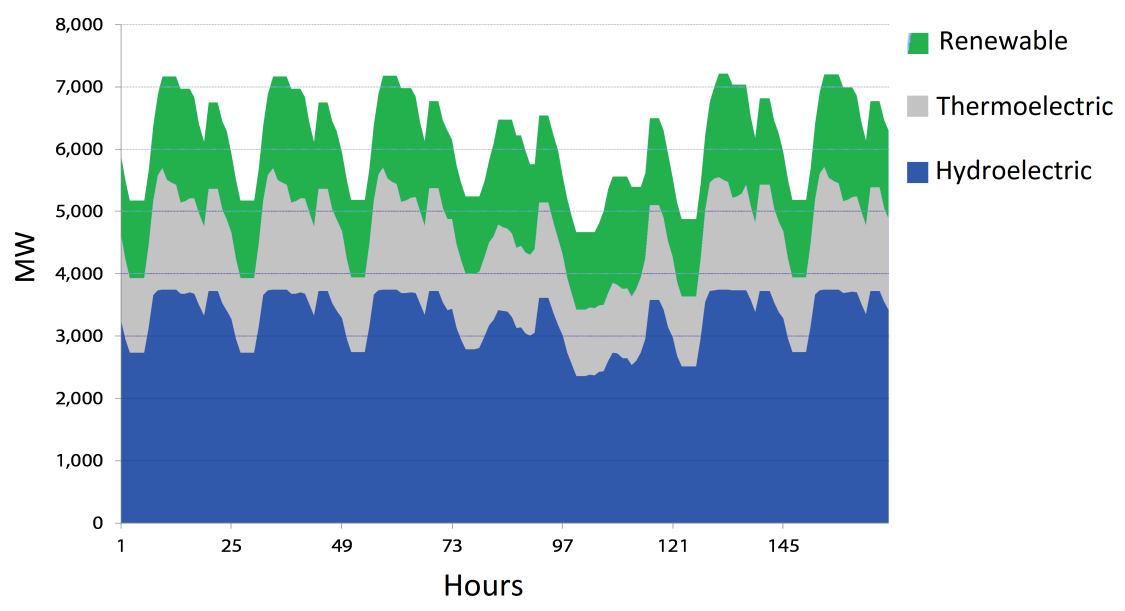

Figure 6.3: Total generation by technology in a typical week

In the case study, 10 of the 54 renewable scenarios were used in the week-ahead planning, and the whole 54 scenarios were used in the simulation phase.

As the current dataset does not have sufficient scenarios to build the uncertainty set, it was necessary to use 54 hourly scenarios of 149 weeks to build the uncertainty set. These scenarios were validated against another set of 16,000 scenarios, a little more than 296 weeks, to validate the decision rule's violation probability.

The uncertainty set was build using the method described in Section (4.2.2), where an optimization problem to build the uncertainty bounds was solved ex-ante, and the uncertainty bounds served as input to the multistage stochastic chance-constrained dispatch. The bounds obtained are displayed at Figures (6.4) and (6.5).

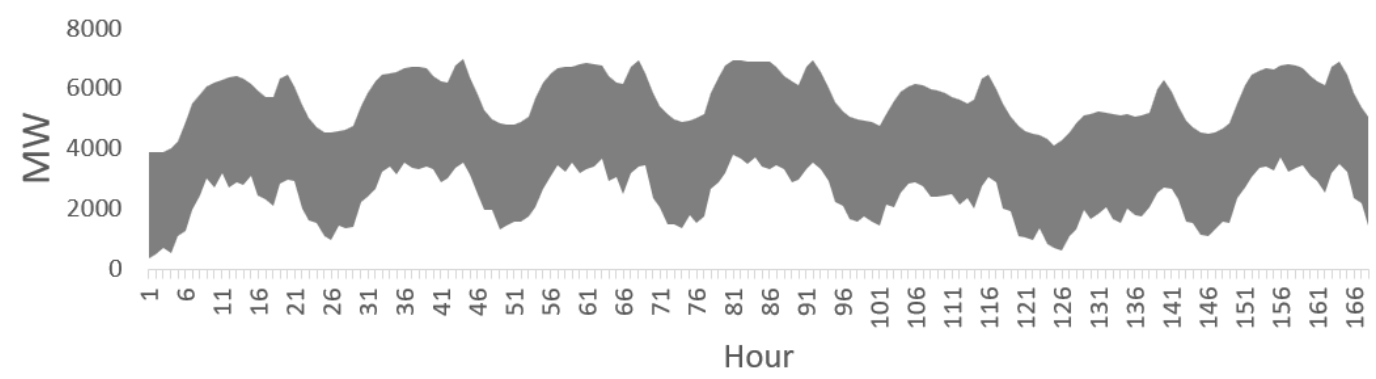

Figure 6.4: SIC system uncertainty bounds 


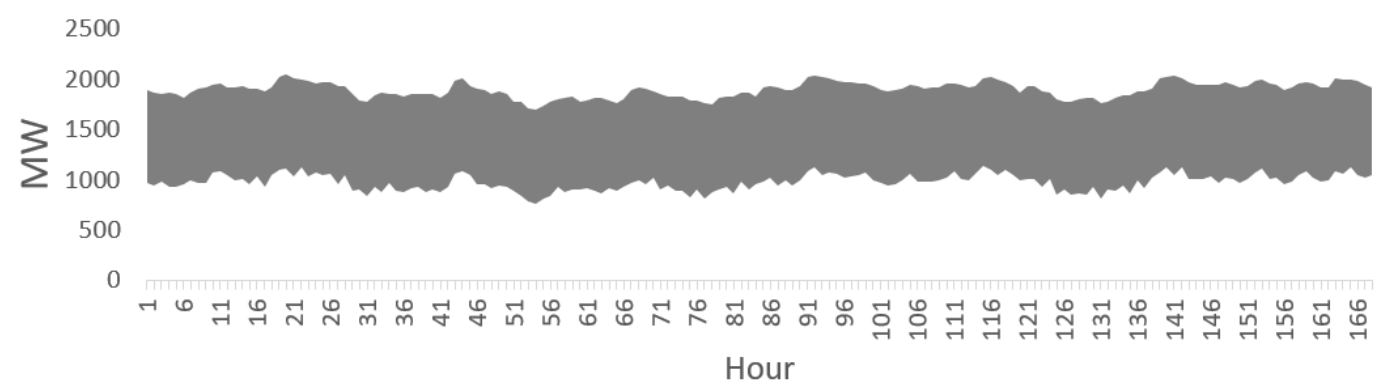

Figure 6.5: SING system uncertainty bounds

\section{2}

\section{Evaluation procedure}

The model evaluation simulates a week-ahead dispatch planning with binding dispatch decisions, for that a two-phase procedure is adopted. The first phase is a week-ahead dispatch planning. The second phase is a "true-up" simulation of the real-time operation, where the system operator applies the dispatch plan to the realized scenario as shown in Figure (6.6).

In the second phase, the dispatch is allowed to deviate from the planned at the cost of the planning phase's spot price. The simulation can compute both the total expected cost and the redispatch cost of the planned decision.

By comparing the simulation cost of the proposed model dispatch with a deterministic and a two-stage stochastic model, it is possible to evaluate our model's benefit to the short-term planning. A schema of the procedure adopted to assess the methodology is shown in Figure (6.7).
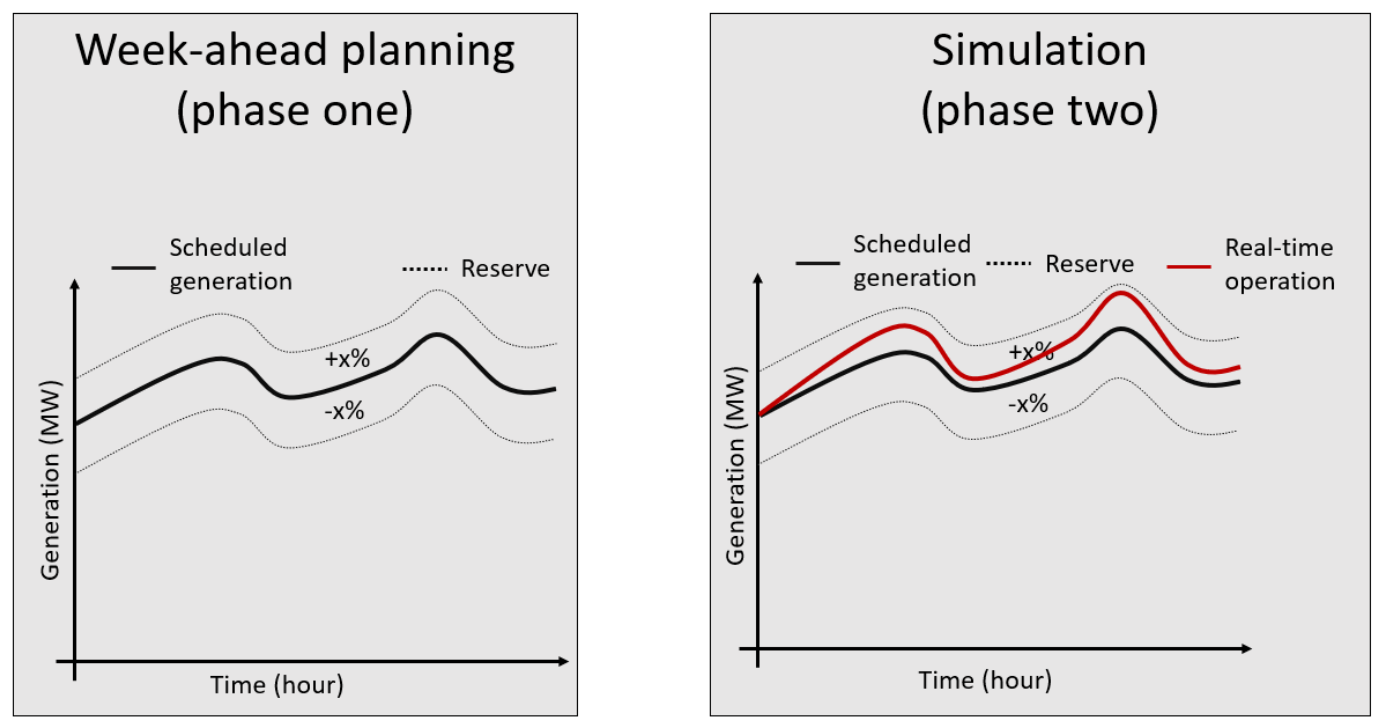

Figure 6.6: Two-phase procedure scheme 


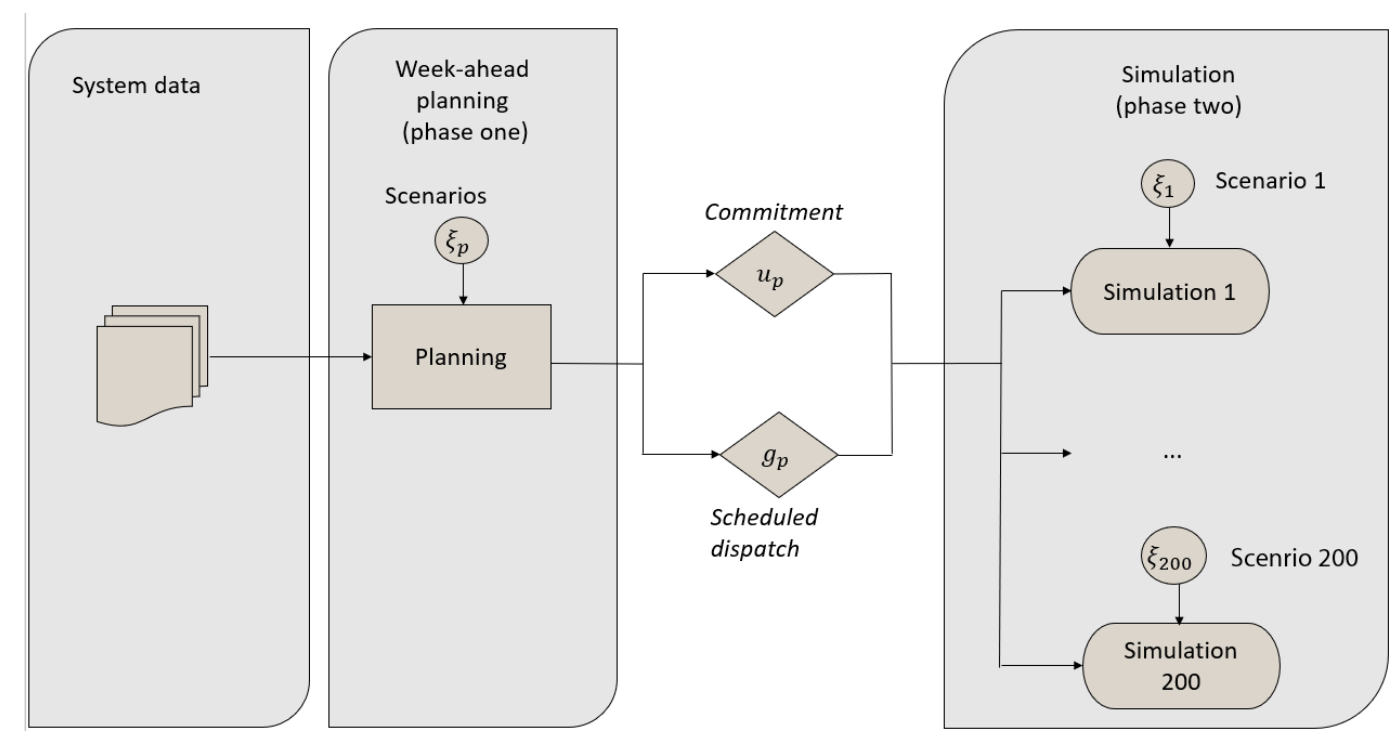

Figure 6.7: Planning procedure

The test considers 54 scenarios of renewable generation, where 10 are used for the week-ahead planning, and 54 ( 44 out-of-sample and 10 in-sample) are used to evaluate the planning's cost. These scenarios represent the uncertainty in the generation forecast. The forecast considered in the deterministic cases was the average of the 10 in-sample scenarios.

Figure 6.8 shows the reference scenario net demand in the blue line and, in gray, the area containing the net demand realization of the 54 scenarios. Figure 6.9 shows the entire week renewable generation per scenario, compared to the total renewable generation of the reference scenario.

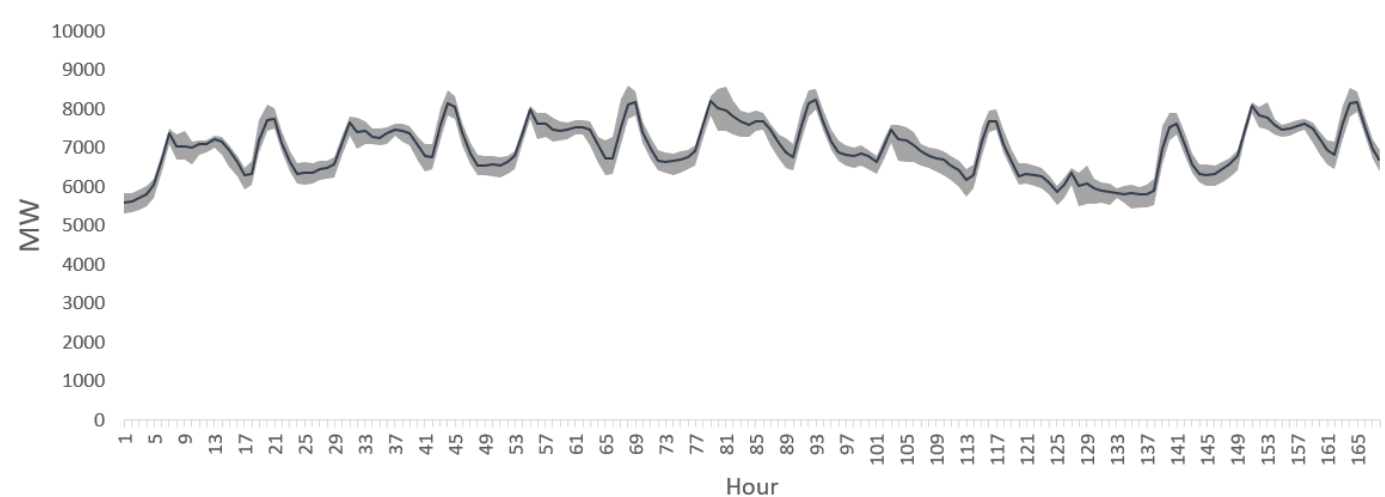

Figure 6.8: Reference scenario and uncertainty region 


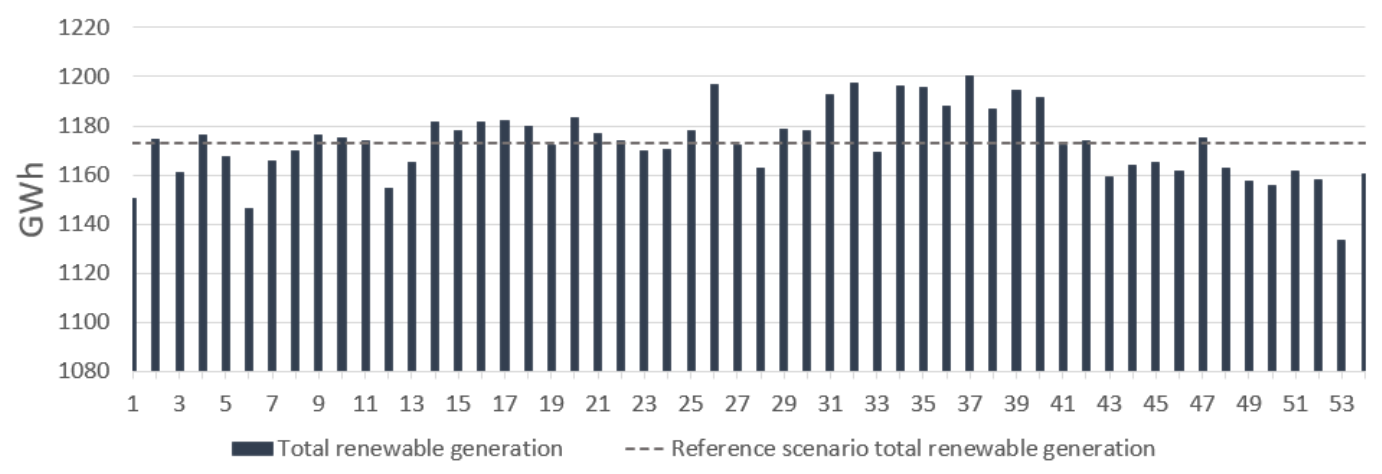

Figure 6.9: Total renewable production by scenario

\section{3}

\section{Model evaluation}

The multistage stochastic model with chance-constraints will be evaluated against three benchmarks. The benchmarks are a deterministic model, a twostage model [62,63], and a multistage affine model.

The models are executed in the Chilean system with two electrical areas, no cluster and without reserve.

Table 6.2: Description of tests cases employed to evaluate the benefit of the chance-constrained model

\begin{tabular}{llccc}
\hline Case ID & Model & Area & Cluster & Reserve \\
\hline T1-DET & Deterministic & 2 & - & No \\
T1-2STAGE & Two-stage stochastic & 2 & - & No \\
T1-MSTAGE & Affine multistage stochastic & 2 & - & No \\
T1-MSTAGE-CPP & Affine multistage stochastic & 2 & - & No \\
& with chance-constraints & & & \\
\hline
\end{tabular}

When comparing the scheduled dispatch, the model T1-MSTAGE-CPP presents more hydro dispatch than the others, as seen in Figure (6.10). Consequently, the thermal dispatch becomes much lower than the other models (6.11).

Figures (6.11) and (6.13) displays the thermal flexibility graph, containing the maximum thermal generation committed capacity (Upwards Flexibility), the minimum thermal generation committed capacity (Downwards Flexibility) and the scheduled generation for each hour. By comparing Figures (6.12) and (6.13), we see that the thermal flexibility of T1-2STAGE resembles much the T1-DET in terms of the range and of modulation. The models T1-MSTAGE and T1-MSTAGE-CPP present a flexibility with the same magnitude but with more variability. 


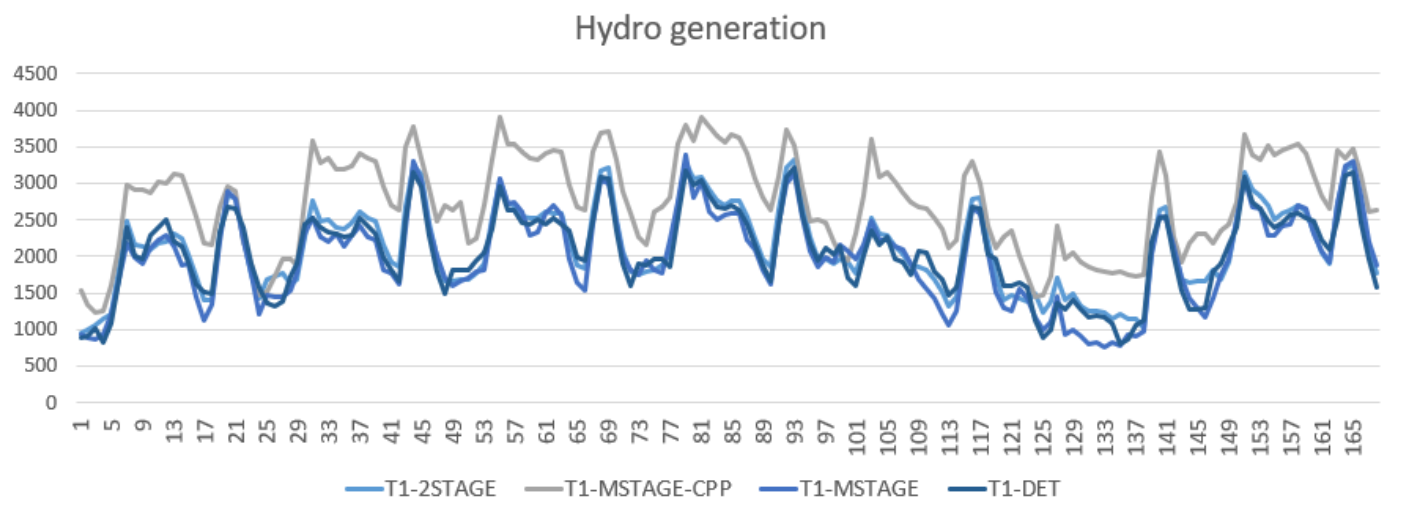

Figure 6.10: Hydro generation

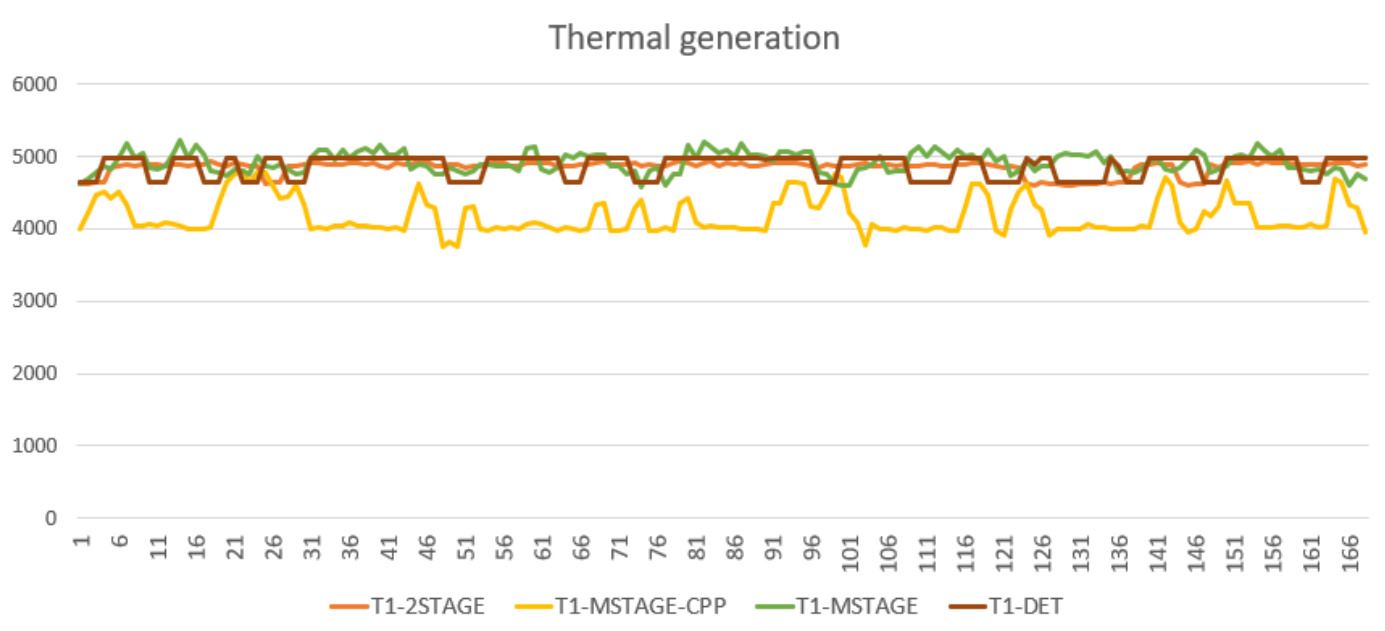

Figure 6.11: Thermal generation

By doing out-of-sample verification of the decision rule feasibility, it is possible to evaluate the Loss of Load probabilities of the multistage models T1-MSTAGE and T1-MSTAGE-CPP. Table (6.3) displays the LOLP obtained by models T1-MSTAGE and T1-MSTAGE-CPP policy, where it is clear the benefit of the chance-constraint by decreasing the LOLP. In this case, the number of scenarios used to evaluate the LOLP was 16,000.

It is worth noticing that although the risk level chosen was of $10 \%$, the results are way more conservative, displaying a LOLP of only $1.75 \%$. The conservativeness is because the methodology proposed by [49] is more conservative in higher-dimensional cases than applying the scenario approach to the original optimization problem.

When it comes to the true-up expected costs, i.e., the out-of-sample expected cost of the dispatch plan, we can see in Figure (6.15) that the T1-DET has the highest cost, followed by the T1-MSTAGE-CCP, T1-MSTAGE, and T1-2STAGE. In the true-up phase, we can see that the T1-2STAGE results 
T1-DET

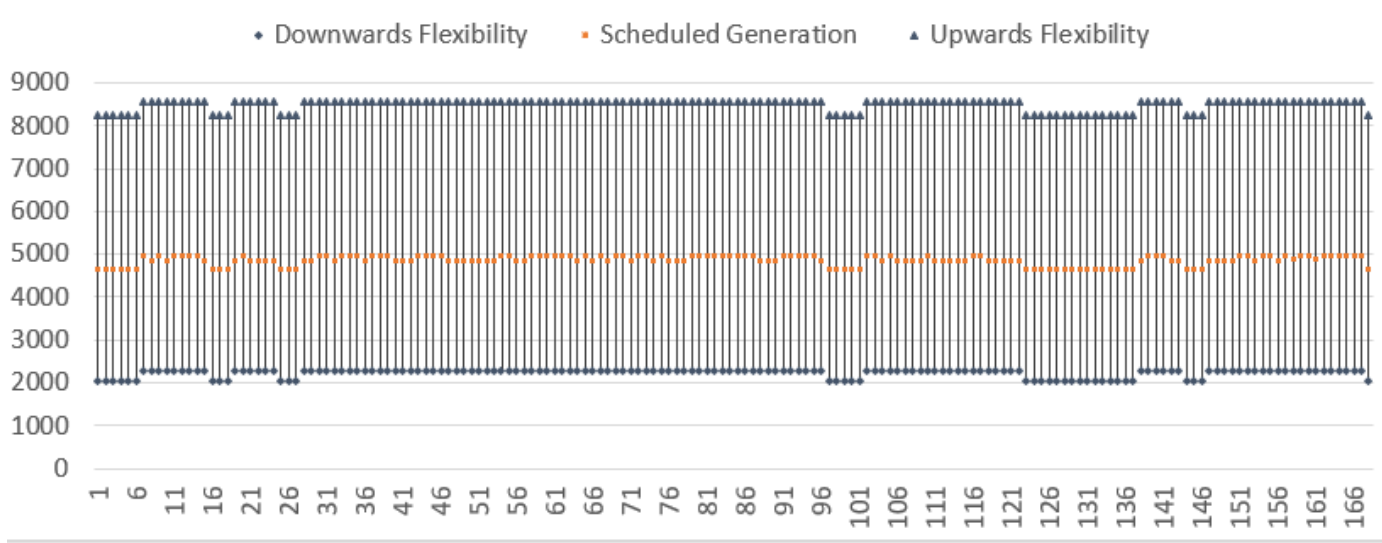

T1-STAGE

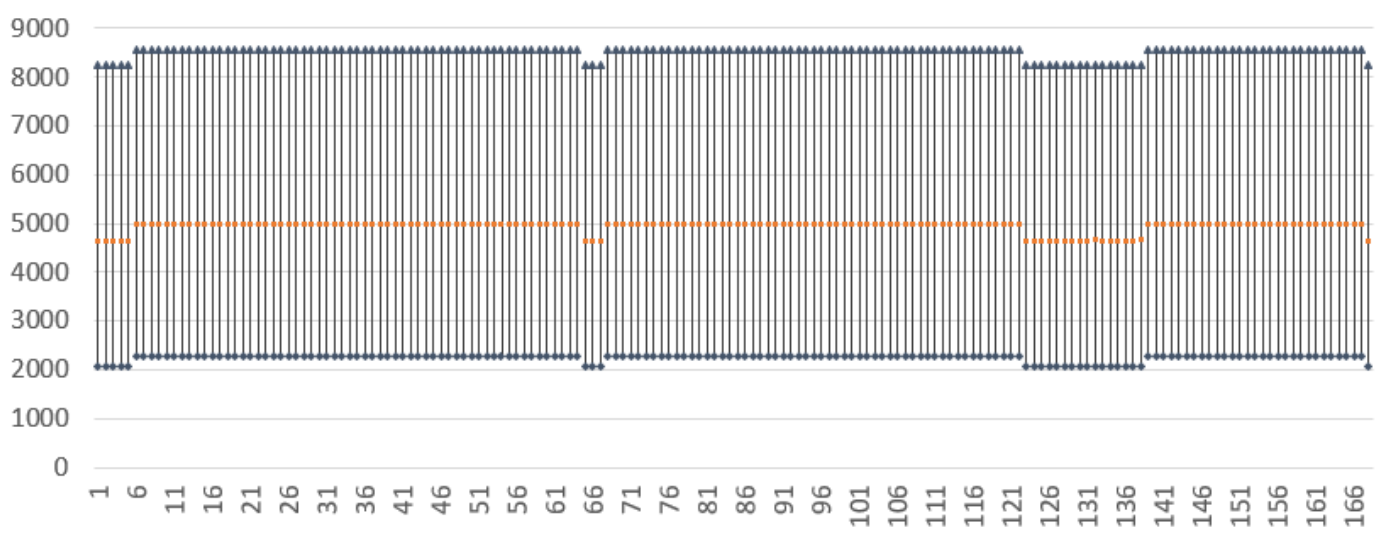

Figure 6.12: Thermal flexibility and scheduled generation for T1-DET and T1-2STAGE

\begin{tabular}{lll}
\hline & T1-MSTAGE-CPP & T1-MSTAGE \\
\hline LOLP & $1.75 \%$ & $66.07 \%$ \\
\hline
\end{tabular}

Table 6.3: Loss of load probability

are the best regarding the other models. Nonetheless, T1-2STAGE is closely followed by T1-MSTAGE, which has an additional advantage representing better the decision-making process, since the model is multistage. The decision rule policy may be improved by applying more flexible decision rules such as the ones described in $[8,10,59]$ or the one proposed in Section (5.3).

The cost savings with respect to the deterministic case, displayed in Figure (6.15), show that the multistage models indeed have a considerable benefit with respect to the T1-DET. The case T1-MSTAGE-CCP presents a lower cost-benefit w.r.t. T1-MSTAGE due to the more restrictive policy, but in turn, it has a much lower violation probability in out-of-sample scenarios. 


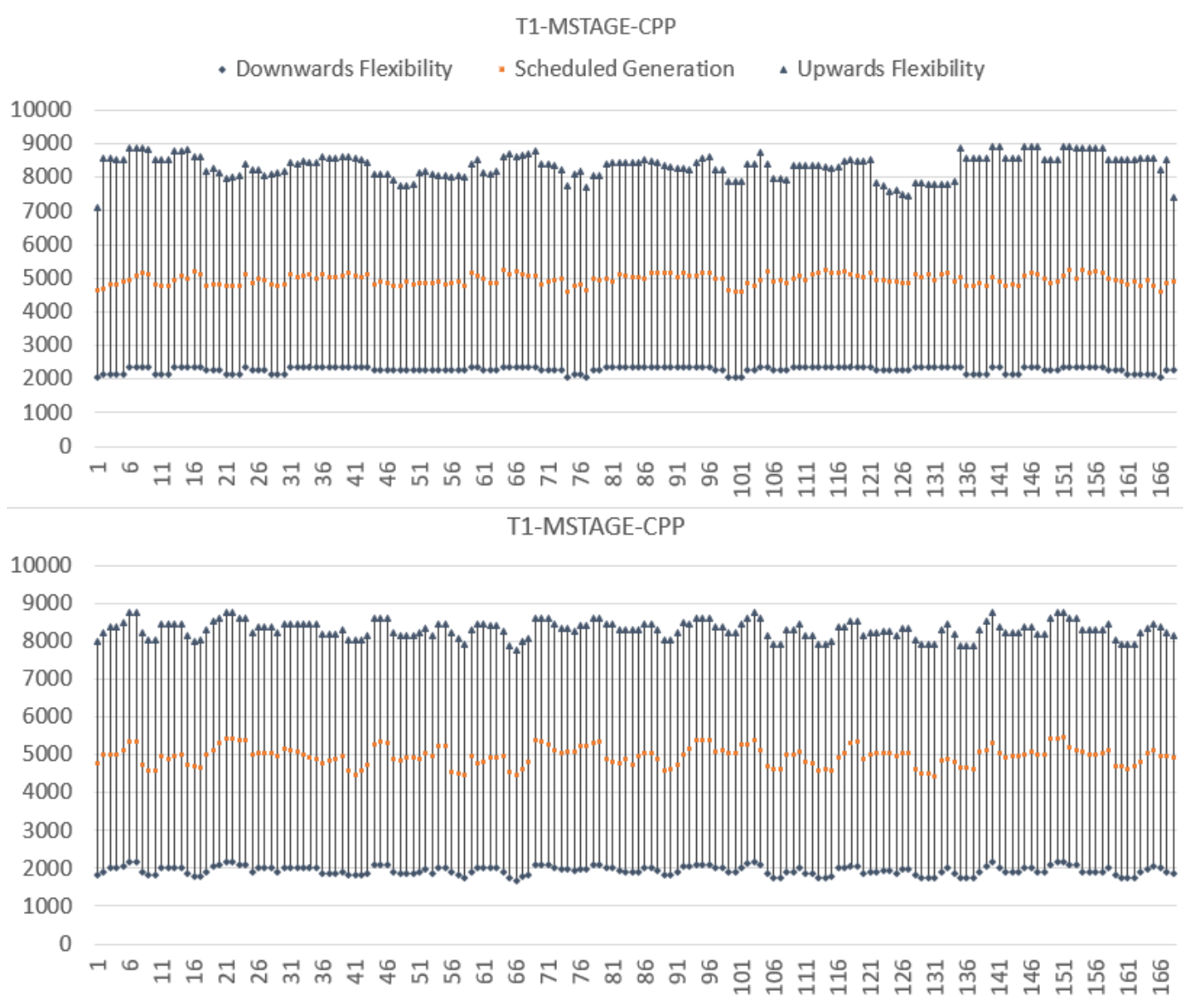

Figure 6.13: Thermal flexibility and scheduled generation for T1-MSTAGE and T1-MSTAGE-CPP

Total expected cost
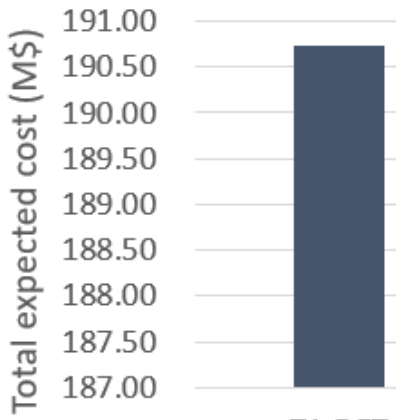

T1-DET
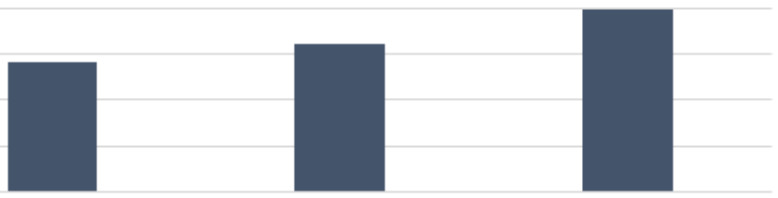

T1-2STAGE

T1-MSTAGE

T1-MSTAGE-CPP

Figure 6.14: Total true-up expected cost 
Total expected saving

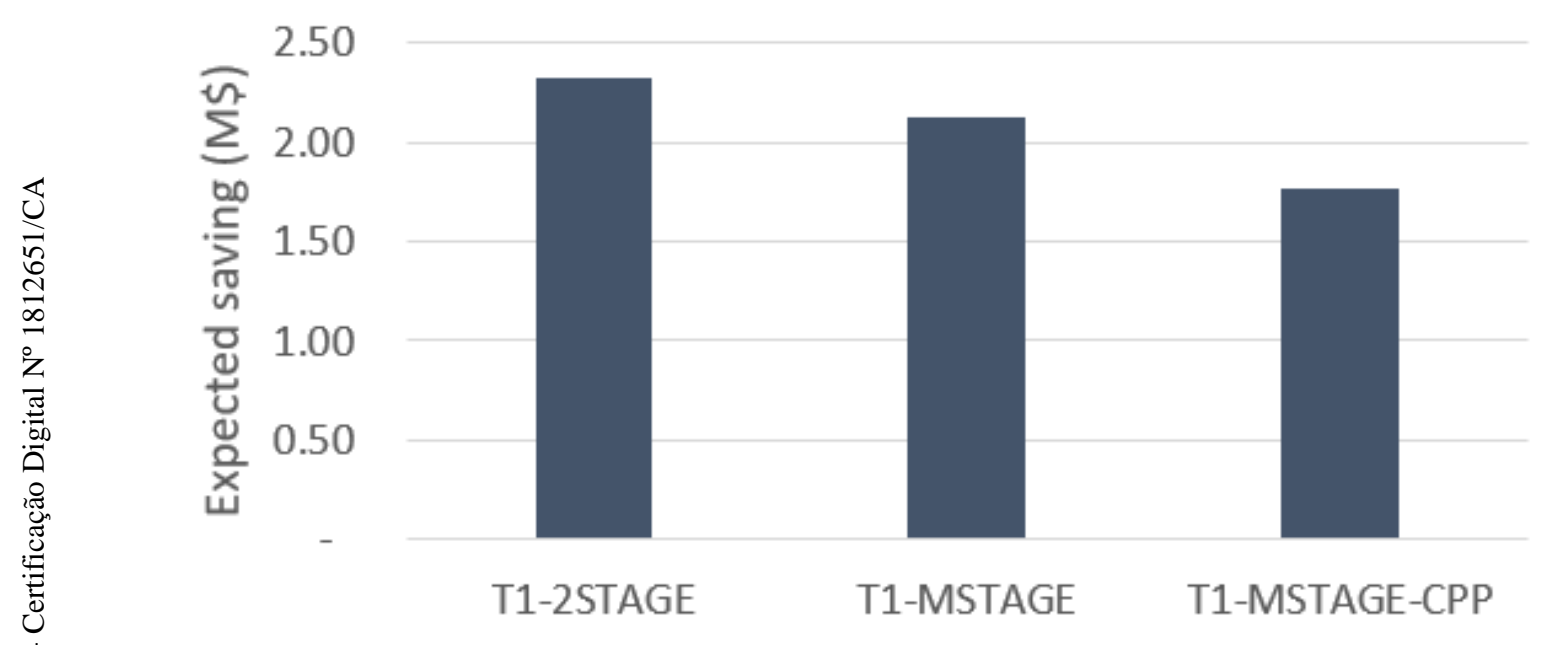

Figure 6.15: Total tru-up expected saving 


\section{4 Reserve requirement performance}

The reserve requirement proposed in Section (5.4) was calculated with the output of the multistage stochastic execution of model T1-MSTAGE-CCP and applied in a deterministic model with reserves. This case is called T2-DET-RES, and the model was evaluated against the cases presented in Table (6.2).

T2-DET-RES was also executed in the Chilean system with two electrical areas and no cluster, as displayed in Table (6.4).

Table 6.4: Description of features in the model T2-DET-RES

\begin{tabular}{llccc}
\hline Case ID & Model & Area & Cluster & Reserve \\
\hline T2-DET-RES & Deterministic & 2 & - & Yes \\
\hline
\end{tabular}

Figure 6.16 shows the reserve requirement obtained from the execution of T1-MSTAGE-CCP. Both hydro and thermal plants are used in reserve, although the hydro plants are used more intensively. It is worth noticing the reserve requirement's dynamic nature, as more reserve requirement is allocated for hours with more uncertainty.

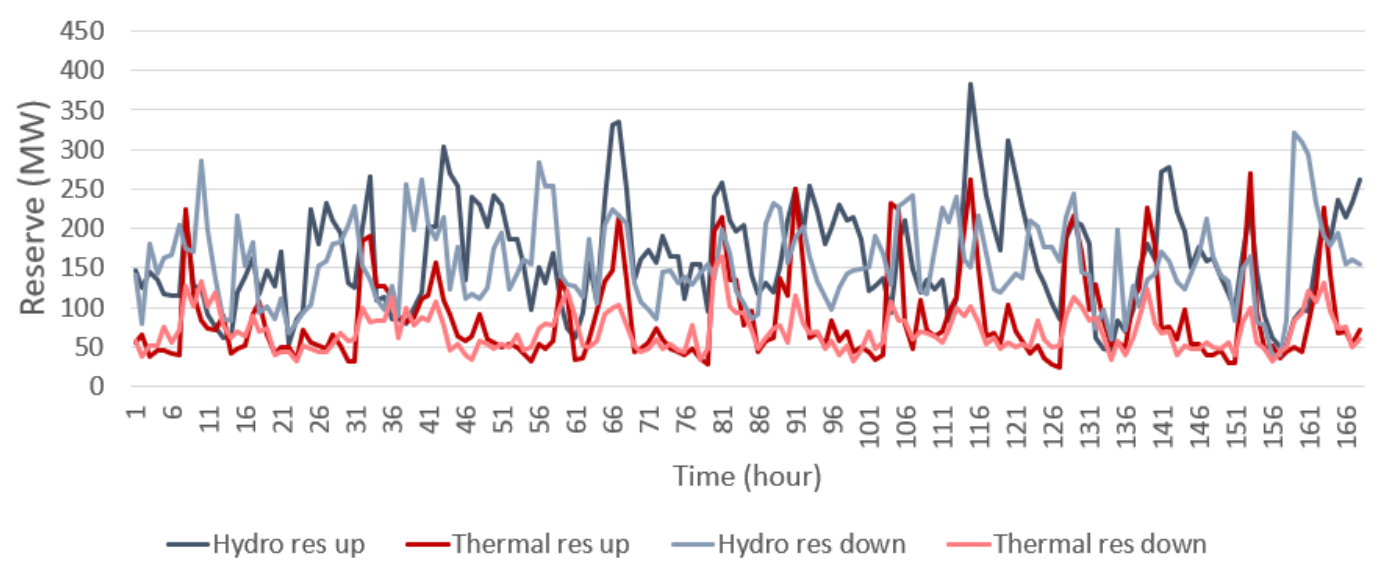

Figure 6.16: Reserve requirement

The thermal flexibility in T2-DET-RES has the same range as T1-DET, but presenting more variability within the hours. This result can be observed by comparing Figure (6.17) and (6.13).

Surprisingly, the model T2-DET-RES was more cost-effective than T1MSTAGE, T1-MSTAGE-CCP, and T1-2STAGE. This result is because the deterministic reserve induces the model correctly to have more conservative behavior in higher uncertainty moments. Furthermore, it does not impose the same level of restrictions of the affine decision rule. This result confirms that 
the affine policy may be too strict and that another more flexible may improve the true-up result.

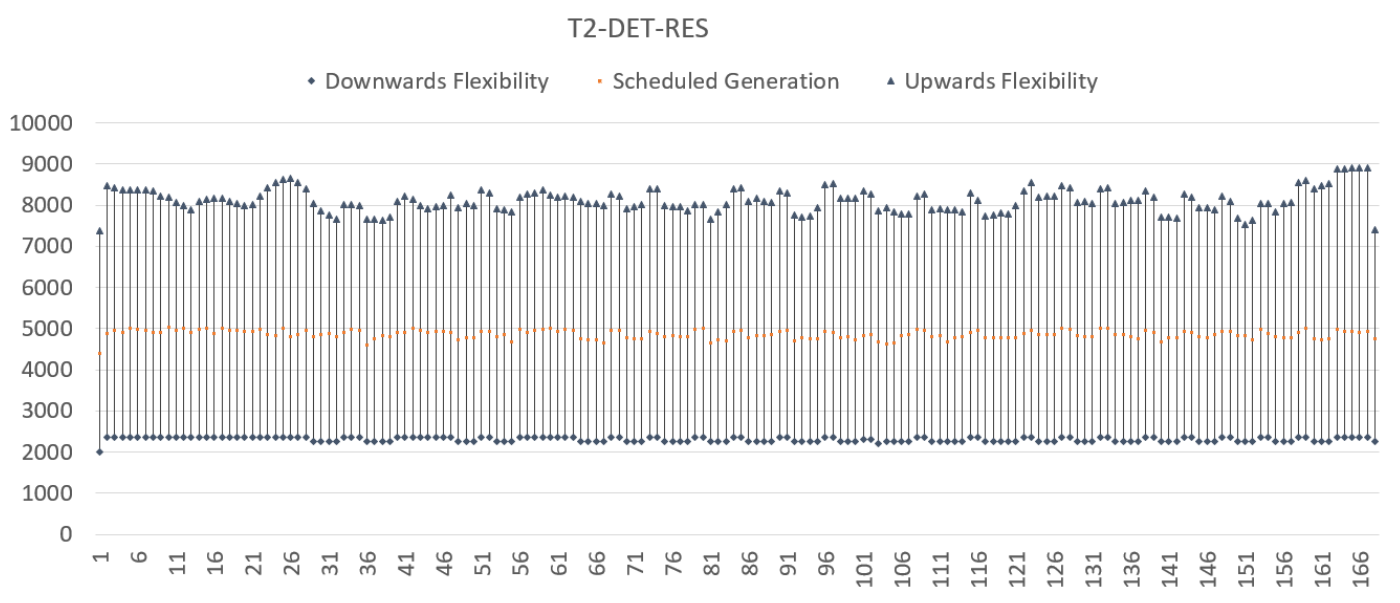

Figure 6.17: Thermal flexibility of T2-DET-RES

Total expected cost

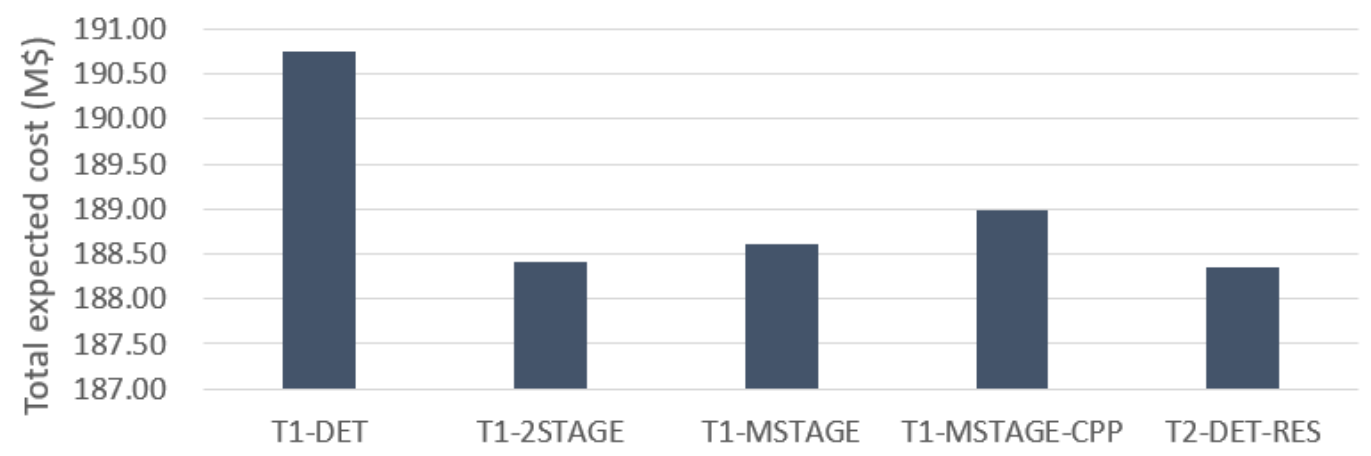

Figure 6.18: Total true-up expected cost 


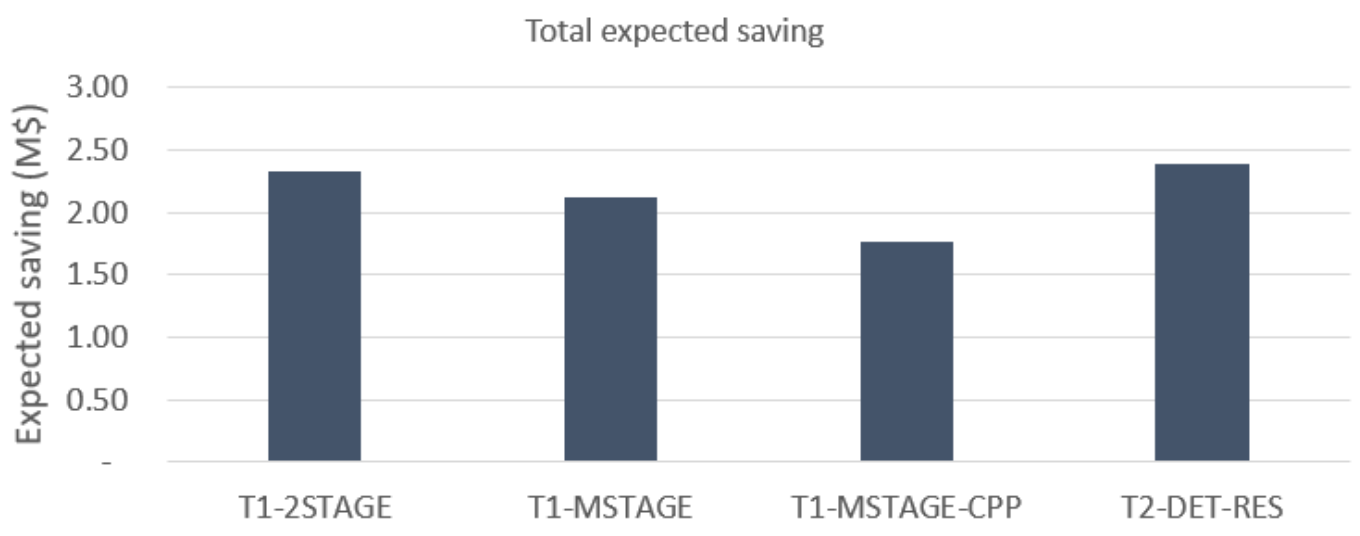

Figure 6.19: Total tru-up expected saving

\section{5}

\section{Improvements impact on the model}

In this Section, the impact in considering electrical areas and scenario clusters are evaluated. Two additional modifications are considered in the model: the first is T3-MSTAGE-1AREA, where a multistage affine model is tested with only one electrical area, and the second is T3-MSTAGE-CLUS, where a multistage affine model is tested with two electrical areas and 3 clusters of scenarios. These models' features are described at Table (6.5) and are evaluated against the cases presented in Table (6.2).

Table 6.5: Description of models T3-MSTAGE-1AREA and T3-MSTAGE-CLUS

\begin{tabular}{llcccc}
\hline Case ID & Model & Area & Cluster & Reserve \\
\hline T3-MSTAGE-1AREA & $\begin{array}{l}\text { Affine } \\
\text { stochastic }\end{array}$ & multistage & 1 & - & No \\
T3-MSTAGE-CLUS & $\begin{array}{l}\text { Affine } \\
\text { stochastic }\end{array}$ & multistage & 2 & 3 & No \\
\hline
\end{tabular}

Considering only one electrical area is another approximation, since the interconnection between SIC and SING is disregarded and thus it is assumed that the power flow in the interconnection does not attains the interconnection limit. With the approximation, the thermal flexibility is increased, and at the same time, the variability is decreased, as seen in Figure (6.20).

Considering scenarios' clusters gives more flexibility to the decision rule since each cluster has a different set of coefficients. By increasing the policy flexibility with clusters in the T3-MSTAGE-CLUS, the flexibility also is slightly increased, with a similar behavior to the T3-MSTAGE-1AREA, which confirms that this feature contributes to the increase of available committed capacity. 


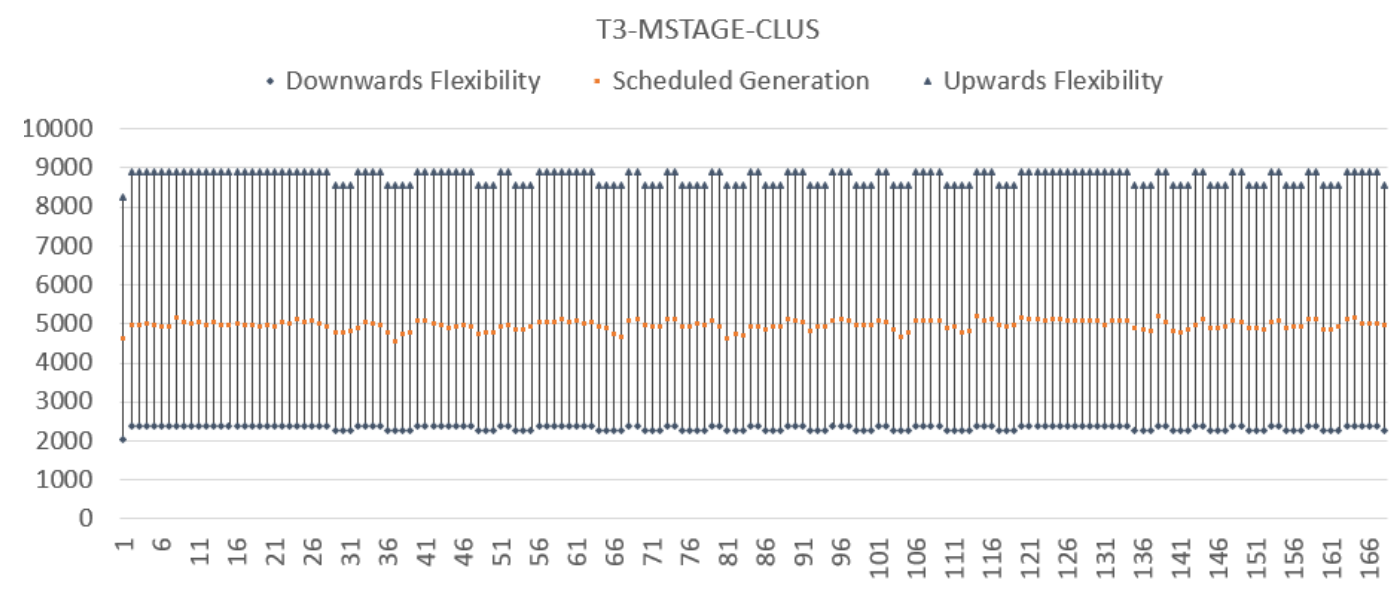

Figure 6.20: Thermal flexibility of T3-MSTAGE-CLUS

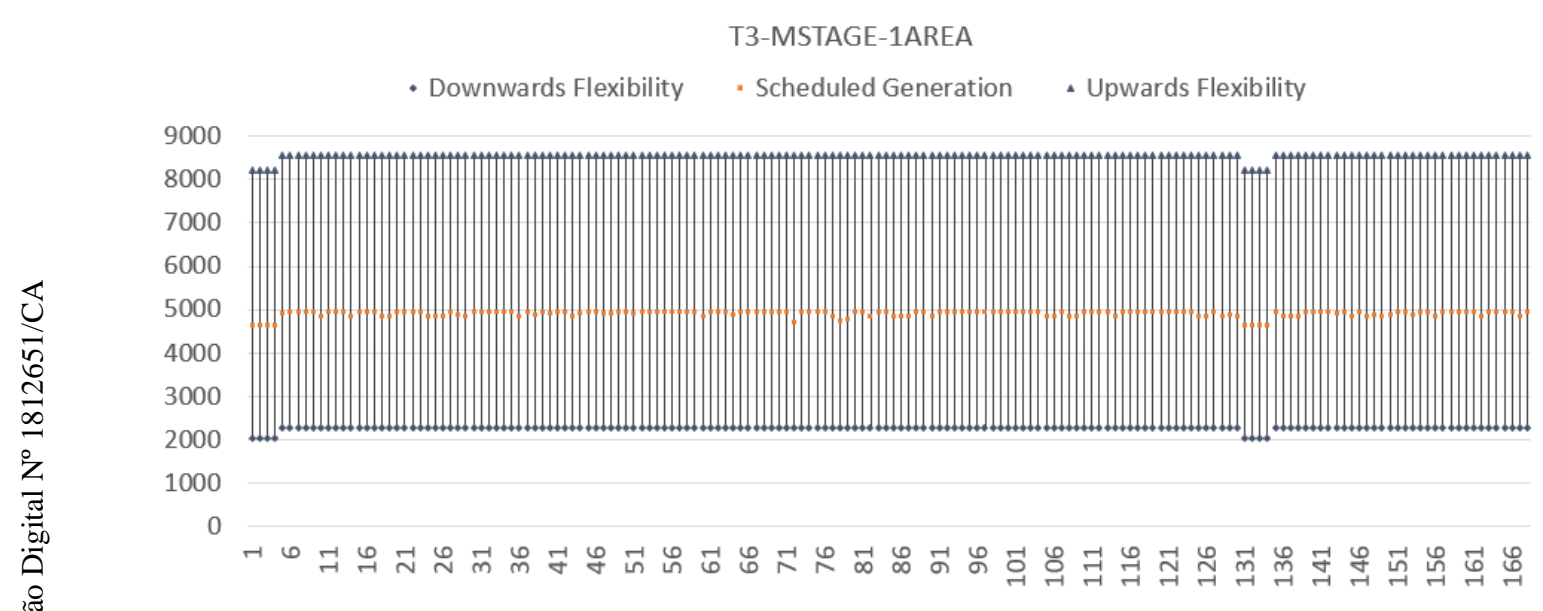

Figure 6.21: Thermal flexibility of T3-MSTAGE-1AREA

As expected, the total cost of both T3-MSTAGE-1AREA and T3MSTAGE-CLUS, seen in Figure (6.22), are lower than T1-MSTAGE, which is accounted respectively for the less constrained optimization problem and the greater flexibility in the affine policy. This result corroborates what was discussed in the previous sections since T3-MSTAGE-CLUS's more flexible affine policy was capable of improving the total expected cost of the T1-MSTAGE and even out-performing the best benchmarks T1-2STAGE and T2-DET. 


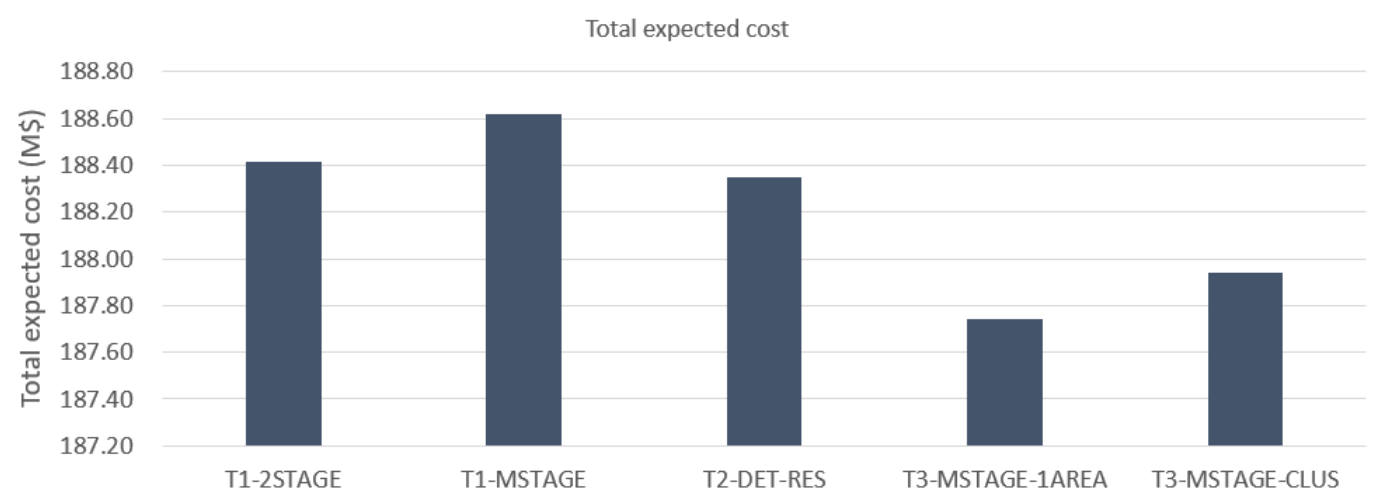

Figure 6.22: Total true-up expected cost

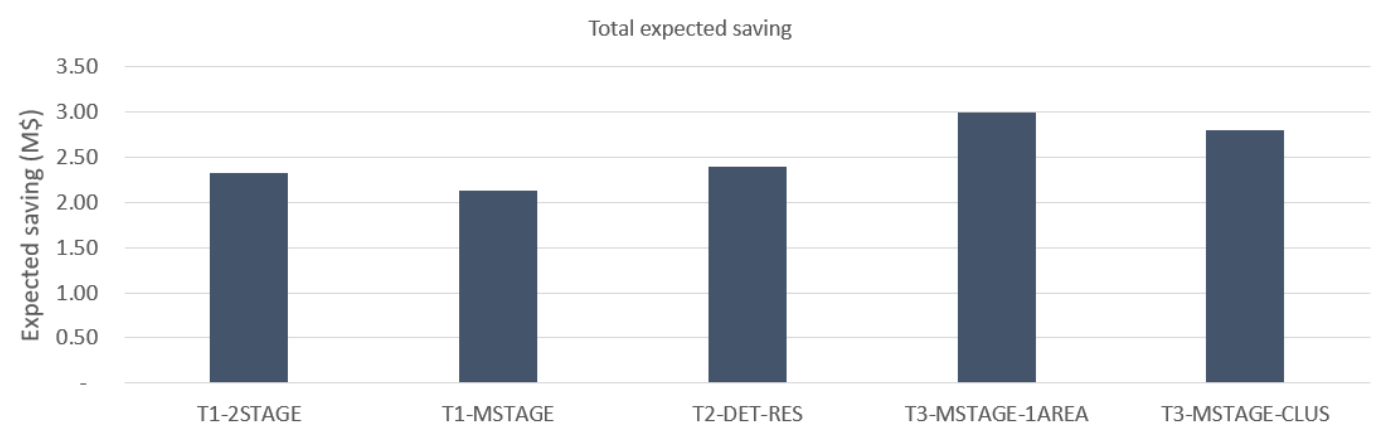

Figure 6.23: Total true-up expected saving

\section{6 \\ Seasonality effect}

Different periods of the year may have different effects on the renewable generation, displaying more or less uncertainty. This test compares the model performance in a different season than the previous sections to assess how the model behaves in a different season of the year.

The net demand scenarios Root-mean-square deviation (RMSD) with respect to the average net demand is evaluated for each month to analyze the seasonality's impact. From Figure (6.24), we can see that the months with the highest RMSD, and therefore highest uncertainty, are January and December, while the lowest is seen in June.

Since the results displayed in Section (6.3) are related to January, this Section will contain only the results of June. By comparing these two results, we can determine the performance under different levels of uncertainty.

Similarly to Section (6.3), this Section contains the results for four different models, displayed in Table (6.6).

Figures (6.25) and (6.26) shows the flexibility of the four test cases, where it shows that the decision rule models T4-MSTAGE and T4-MSTAGE-CCP 


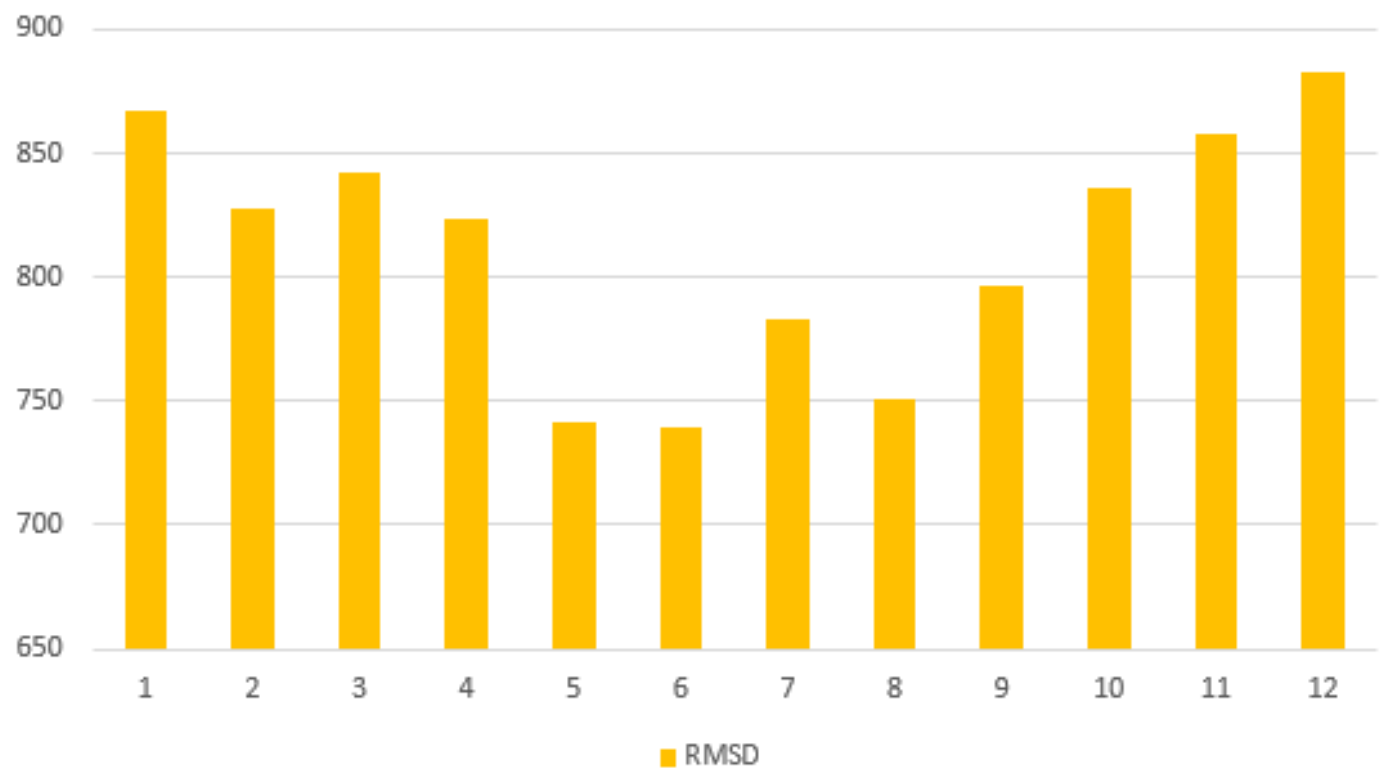

Figure 6.24: Net demand's Root-mean-square deviation per month

Table 6.6: Description of tests cases employed to evaluate the seasonality impact

\begin{tabular}{llccc}
\hline Case ID & Model & Area & Cluster & Reserve \\
\hline T4-DET & Deterministic & 2 & - & No \\
T4-2STAGE & Two-stage stochastic & 2 & - & No \\
T4-MSTAGE & Affine multistage stochastic & 2 & - & No \\
T4-MSTAGE-CPP & Affine multistage stochastic & 2 & - & No \\
& with chance-constraints & & & \\
\hline
\end{tabular}

have more variability on the committed thermal capacity than models T4-DET and T4-MSTAGE, which presents a flat profile of committed thermal capacity.

The total cost of the models T4-MSTAGE and T4-MSTAGE-CCP are still lower than the model T4-DET, showing a significant benefit. Nonetheless, the model T4-2STAGE has a total expected cost significantly lower than the other models, also outperforming them in the months with lower variability. By comparing the performance of the multistage models and the two-stage model in both scenarios, with high and low variability, it is possible to infer by the total expected saving that difference in performance from the two-stage model to the others is greater with low variability scenarios.

The lower variability of June's renewable scenarios decreased the outof-sample LOLP of T4-MSTAGE-CPP to only $0.38 \%$, while increased the LOLP of T4-MSTAGE as seen in Table (6.6). This results shows that when the chance-constraint is not considered in the planning, nothing can be said about the feasibility of this policy, since the out-of-sample feasibility of the decision 
T4-DET

- Downwards Flexibility ॥ Scheduled Generation \&Upwards Flexibility

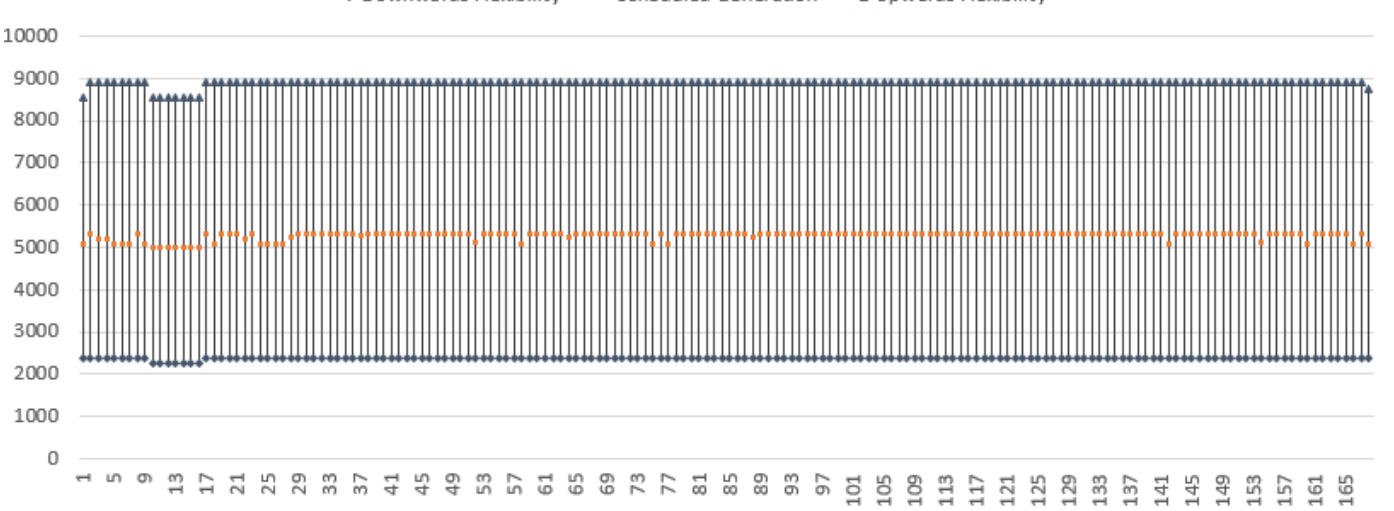

T4-2STAGE

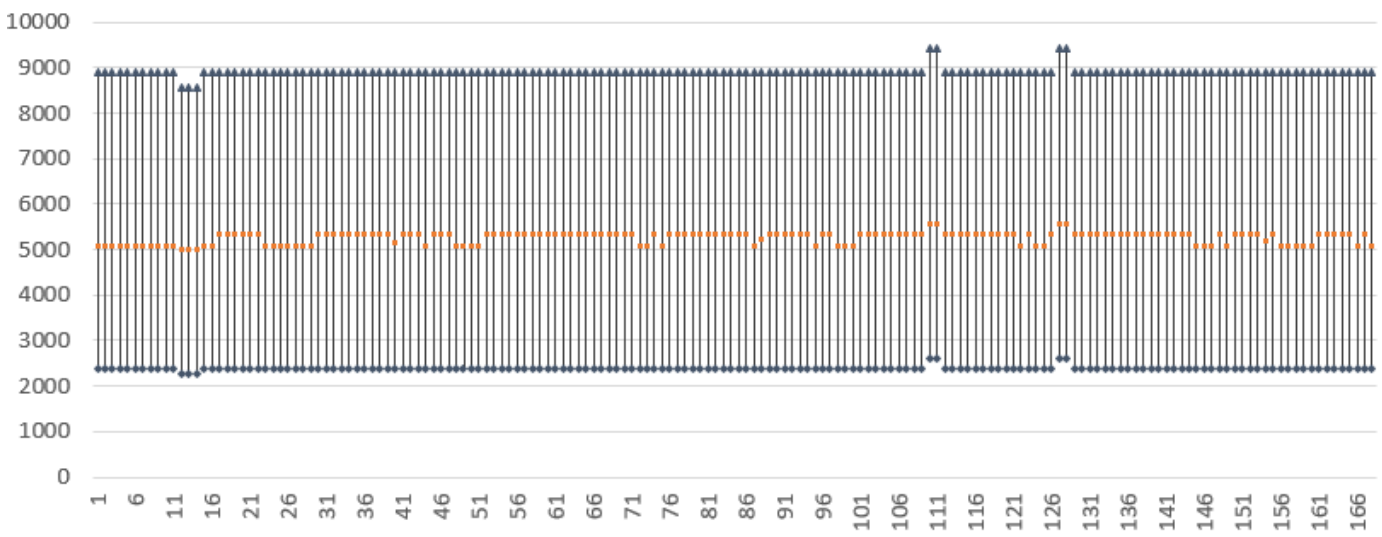

Figure 6.25: Thermal flexibility and scheduled generation for T4-DET and T4-2STAGE

\begin{tabular}{lll}
\hline & T4-MSTAGE-CPP & T4-MSTAGE \\
\hline LOLP & $0.38 \%$ & $85.55 \%$ \\
\hline
\end{tabular}

Table 6.7: Loss of load probability

rule is not guaranteed and may present unacceptable results. 


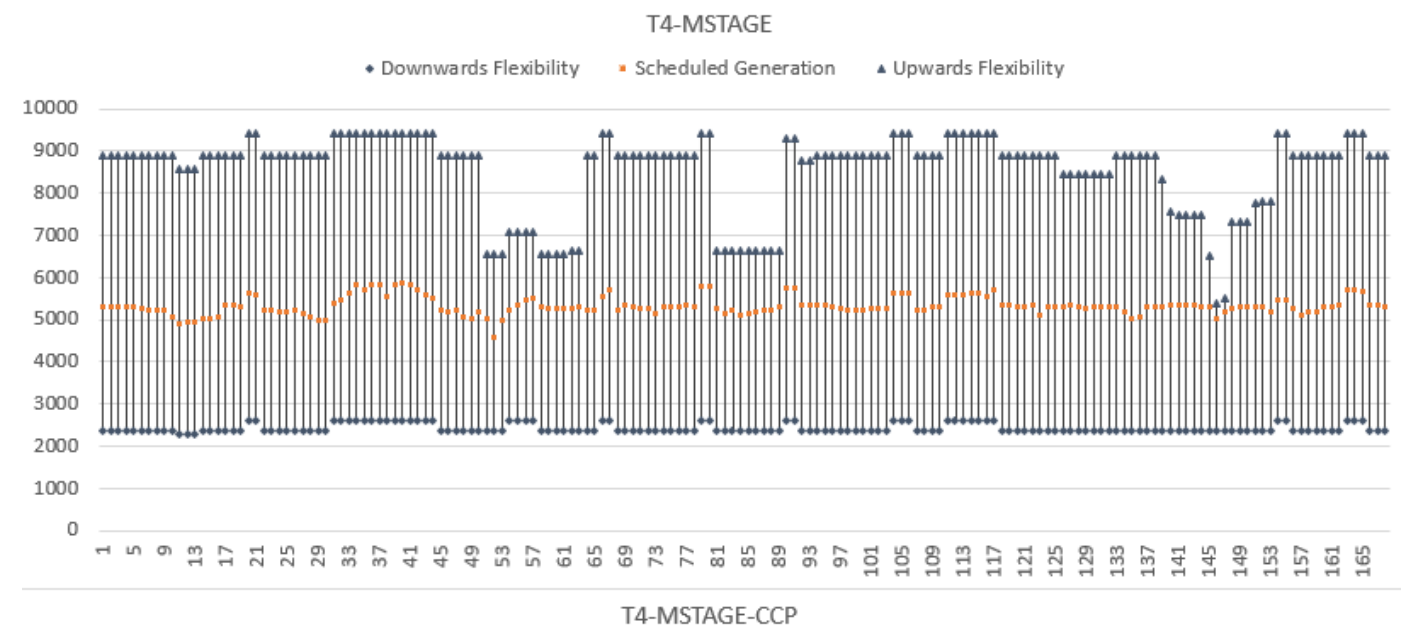

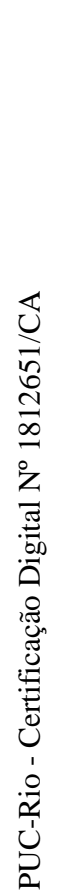

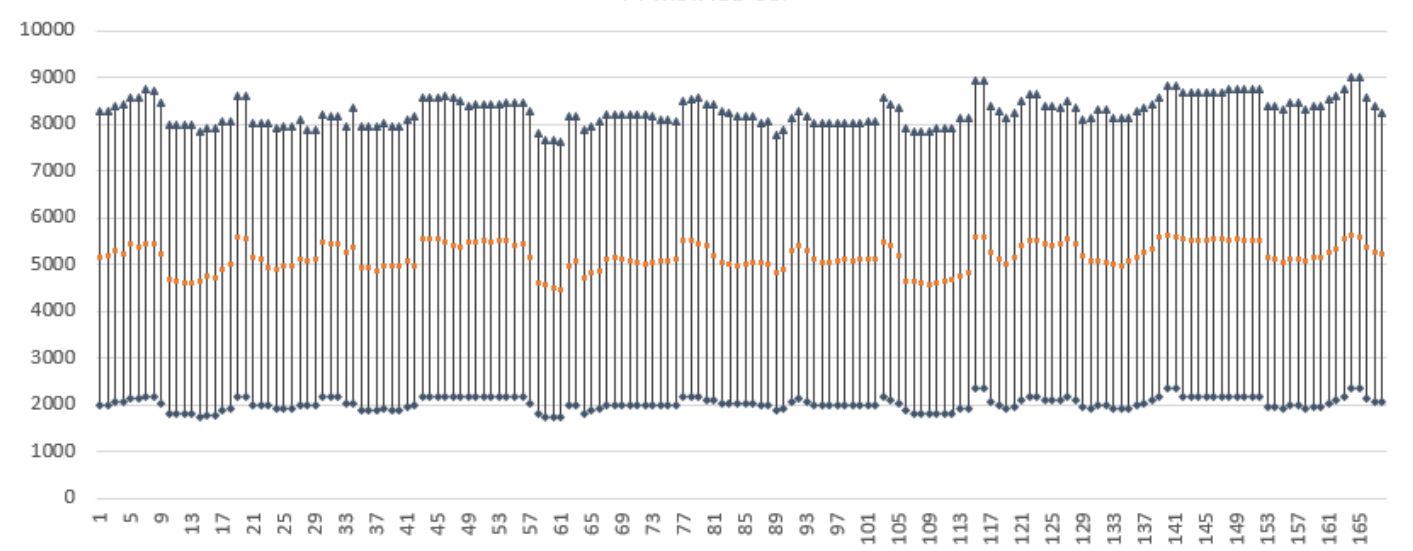

Figure 6.26: Thermal flexibility and scheduled generation for T4-MSTAGE and T4-MSTAGE-CCP

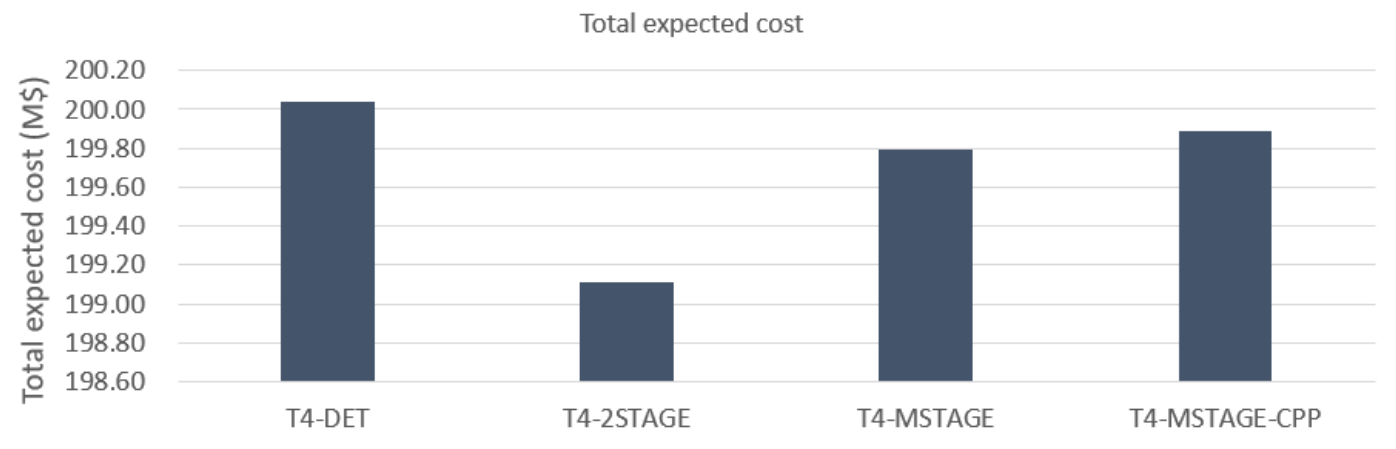

Figure 6.27: Total true-up expected cost 
Total expected saving

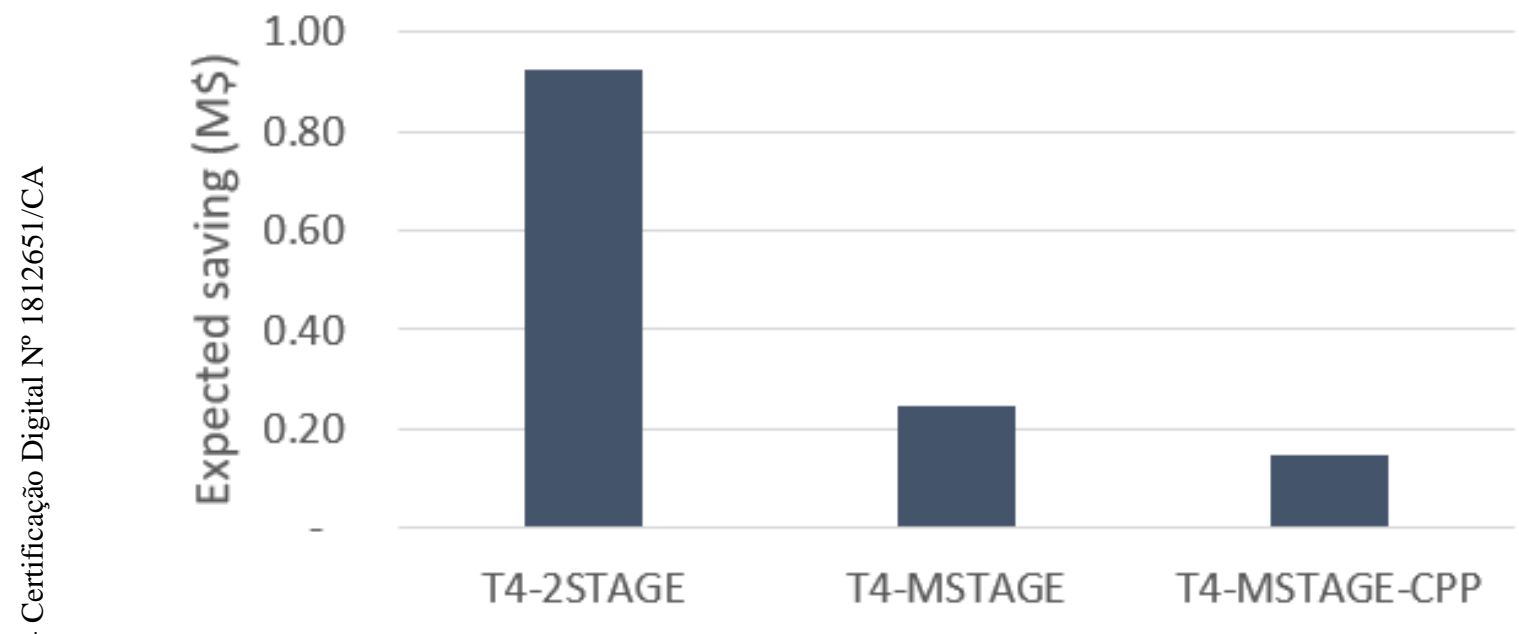

Figure 6.28: Total true-up expected saving 


\section{Conclusion}

This work presented a multistage stochastic formulation of the weak-ahead scheduling problem with chance-constraints. The weak-ahead problem has both commitment and reservoir time-coupling constraints. The chance-constraints guarantee that the load balance is supplied with any desired probability level, and it was used the scenario approach to solve the chance-constraint.

Applying joint chance-constraints to a multistage stochastic unitcommitment model is very defying, since separately a joint chance-constrained problem and a multistage stochastic unit-commitment problem are already challenging. This work proposes a methodology to apply both techniques in an acceptable computational time with a data-driven approach to the chanceconstraints.

To make the problem computationally tractable, an affine decision rule simplification to the stochastic problem was made, and a novel affine formulation is proposed. This formulation gives physical meaning to the affine translation coefficient and has a synergy with the approach used to tackle the joint chanceconstraint.

This work adopted the scenario approach methodology to solve the chanceconstraint. The scenario approach is known to have a major downside that is the great number of constraints. In this work, the latest developments in the scenario approach theory were evaluated, and the methodology proposed by Margellos et al. [6] was the best fit since it did not incur much computational time to the unit-commitment problem, as the scenario approach problem was solved ex-ante.

The proposed multistage affine stochastic model and all its proposed variants were tested in the Chilean system. It became clear the benefit of the proposed formulation against both a deterministic and a two-stage model. The stochastic formulation with clusters of decision rule presented the lowest expected cost because the uncertainty is explicitly considered in the multistage planning model.

The proposed reserve requirement method's performance also displayed a great result, showing to be very effective as its performance was better than that of a two-stage model. The proposed reserve requirement is probabilistic 
and dynamic, as it allocates the same risk level for each hour.

The joint chance-constraints showed to be effective in reducing the LOLP, which was reduced to only $0.125 \%$ in out-of-sample scenarios. The synergy between the decision rule and the scenario approach methodology was exploited to create "feasibility cuts", which are cuts that ensure the feasibility of a decision rule under a probability level. Furthermore, these cuts also ensure the LOLP is attained since the affine unit-commitment model uses the net demand uncertainty as a decision rule parameter. 


\section{Bibliography}

[1] SCHILDBACH, G.; FAGIANO, L. ; MORARI, M.. RANDOMIZED SOLUTIONS TO CONVEX PROGRAMS WITH MULTIPLE CHANCE CONSTRAINTS. SIAM J. OPTIM, 23(4):2479-2501, 2013.

[2] VAN ACKOOIJ, W.; LOPEZ, I. D.; FRANGIONI, A.; LACALANDRA, F. ; TAHANAN, M.. Large-scale unit commitment under uncertainty: an updated literature survey. Annals of Operations Research, 2018.

[3] CALAFIORE, G.; CAMPI, M.. Uncertain convex programs: randomized solutions and confidence levels. Mathematical Programming, 102(1):25-46, jan 2005.

[4] DUPAČOVÁ, J.; CONSIGLI, G. ; WALLACE, S. W.. Scenarios for Multistage Stochastic Programs. Annals of Operations Research, 100(14):25-53, 2000.

[5] CAMPI, M. C.; GARATTI, S.. A Sampling-and-Discarding Approach to Chance-Constrained Optimization: Feasibility and Optimality. Journal of Optimization Theory and Applications, 148(2):257-280, 2011.

[6] MARGELLOS, K.; GOULART, P. ; LYGEROS, J.. On the road between robust optimization and the scenario approach for chance constrained optimization problems. IEEE Transactions on Automatic Control, 59(8):2258-2263, 2014.

[7] GARSTKA, S. J.; WETS, R. J. B.. On decision rules in stochastic programming. Mathematical Programming, 7(1):117-143, 1974.

[8] CHEN, X.; SIM, M.; SUN, P. ; JIAWEI, Z.. A Linear Decision-Based Approximation Approach to Stochastic Programming. Operations Research, 56(2):344-357, 2008.

[9] CHEN, X.; ZHANG, Y.. Uncertain Linear Programs : Extended Affinely Adjustable Robust Counterparts. Operations Research, 2009.

[10] GEORGHIOU, A.; WIESEMANN, W. ; KUHN, D.. Generalized decision rule approximations for stochastic programming via liftings. Math. Program., Ser. A, 2014. 
[11] KUHN, D.; WIESEMANN, W. ; GEORGHIOU, A.. Primal and dual linear decision rules in stochastic and robust optimization. Mathematical Programming, 130(1):177-209, 2011.

[12] SHAPIRO, A.; NEMIROVSKI, A.. On complexity of stochastic programming problems. Optimization-online, p. 111-146, 2006.

[13] BEN-TAL, A.; GORYASHKO, A.; GUSLITZER, E. ; NEMIROVSKI, A.. Adjustable robust solutions of uncertain linear programs. Math. Program., Ser. A, 99:351-376, 2004.

[14] CALAFIORE, G. C.. Multi-period portfolio optimization with linear control policies. Automatica, 44(10):2463-2473, 2008.

[15] LORCA, Á.; SUN, X. A.; LITVINOV, E. ; ZHENG, T.. Multistage adaptive robust optimization for the unit commitment problem. Operations Research, 64(1):32-51, 2016.

[16] WARRINGTON, J.; GOULART, P. J.; MARI, S. ; MORARI, M.. Robust reserve operation in power systems using affine policies. 51st IEEE Conf. Decision and Control, p. 1111-1117, 2012.

[17] WARRINGTON, J.; MEMBER, S.; GOULART, P.; MARIÉTHOZ, S. ; MORARI, M.. Policy-Based Reserves for Power Systems. IEEE Transactions on Power Systems, 2013.

[18] WARRINGTON, J.; HOHL, C.; GOULART, P. J. ; MORARI, M.. Optimal unit commitment accounting for robust affine reserve policies. 2014 American Control Conference (ACC), 2014.

[19] JABR, R. A.. Adjustable Robust OPF With Renewable Energy Sources. IEEE Transactions on Power Systems, 28(4):4742 - 4751, 2013.

[20] JABR, R. A.. Linear Decision Rules for Control of Reactive Power by Distributed Photovoltaic Generators. IEEE Transactions on Power Systems, 33(2):2165-2174, 2018.

[21] EGGING, R.; FLETEN, S. E.; GRØNVIK, I.; HADZIOMEROVIC, A. ; INGVOLDSTAD, N.. Linear decision rules for hydropower scheduling under uncertainty. IEEE Transactions on Power Systems, 32(1):103-113, 2017.

[22] CALAFIORE, G. C.. RANDOM CONVEX PROGRAMS. SIAM J. OPTIM, 20(6):3427-3464, 2010. 
[23] ZHANG, X.; GRAMMATICO, S.; SCHILDBACH, G.; GOULART, P. ; LYGEROS, J.. On the sample size of randomized MPC for chanceconstrained systems with application to building climate control. 2014 European Control Conference, ECC 2014, p. 478-483, 2014.

[24] CHARNES, A.; COOPER, W. W. ; SYMONDS, G. H.. Cost horizons and certainty equivalents: An approach to stochastic programming of heating oil. Management Science, 4(3):235-263, 1958.

[25] PAGNONCELLI, B. K.; AHMED, S. ; SHAPIRO, A.. Sample average approximation method for chance constrained programming: Theory and applications. Journal of Optimization Theory and Applications, 142(2):399-416, 2009.

[26] NEMIROVSKI, A.; SHAPIRO, A.. Convex approximations of chance constrained programs. SIAM Journal on Optimization, 17(4):959-996, 2006.

[27] LUEDTKE, J.; AHMED, S.. A sample approximation approach for optimization with probabilistic constraints. SIAM Journal on Optimization, 19(2):674-699, 2008.

[28] CALAFIORE, G. C.; CAMPI, M. C.. The scenario approach to robust control design. IEEE Transactions on Automatic Control, 51(5):742-753, 2006.

[29] CAMPI, M. C.; GARATTI, S.. THE EXACT FEASIBILITY OF RANDOMIZED SOLUTIONS OF UNCERTAIN CONVEX PROGRAMS. SIAM J. OPTIM, 19(3):1211-1230, 2008.

[30] ZHANG, X.; GRAMMATICO, S.; SCHILDBACH, G.; GOULART, P. ; LYGEROS, J.. On the sample size of random convex programs with structured dependence on the uncertainty. Automatica, 60:182-188, 2015.

[31] RUDIN, W.; RUDIN, W. ; COMPANY, T. M.-H. P.. Real and Complex Analysis. Higher Mathematics Series. McGraw-Hill Education, 1987.

[32] ZHENG, Q. P.; WANG, J.; MEMBER, S. ; LIU, A. L.. Stochastic Optimization for Unit Commitment - A Review. IEEE Transactions on Power Systems, p. 1-12, 2014.

[33] RUIZ, P.; PHILBRICK, C.; ZAK, E.; CHEUNG, K. ; SAUER, P.. Uncertainty management in the unit commitment problem. IEEE Transactions on Power Systems, 24(2):642-651, 2009. 
[34] TAHANAN, M.; VAN ACKOOIJ, W.; FRANGIONI, A. ; LACALANDRA, F.. Large-scale Unit Commitment under uncertainty. 4or, 13(2):115171, 2015.

[35] KEYHANI, A.; MARWALI, M. ; DAI, M.. Integration of green and renewable energy in electric power systems. Wiley, 2010.

[36] WANG, Q.; GUAN, Y. ; WANG, J.. A chance-constrained two-stage stochastic program for unit commitment with uncertain wind power output. IEEE Transactions on Power Systems, 27(1):206-215, 2012.

[37] ZOU, J.; AHMED, S. ; SUN, A.. Multistage stochastic unit commitment using stochastic dual dynamic integer programming. IEEE Transactions on Power Systems, 34:1814-1823, 2019.

[38] LUEDTKE, J.; AHMED, S. ; NEMHAUSER, G. L.. An integer programming approach for linear programs with probabilistic constraints. Mathematical Programming, 122:247-272, 42010.

[39] QIU, F.; AHMED, S.; DEY, S. S. ; WOLSEY, L. A.. Covering linear programming with violations. INFORMS Journal on Computing, 26:531546, 2014.

[40] CARPEnTIER, P.; GOHEN, G.; CULIOLI, J. ; RENAUD, A.. Stochastic optimization of unit; commitment: a new decomposition framework. IEEE Transactions on Power System, 11:1067-1073, 1996.

[41] KIWIEL, K. C.. An aggregate subgradient method for nonsmooth and nonconvex minimization. Journal of Computational and Applied Mathematics, 14:391-400, 1986.

[42] TAKRITI, S.; KRASENBRINK, B. ; WU, L. S.-Y.. Incorporating fuel constraints and electricity spot prices into the stochastic unit. Operations Research, 48:268-280, 2000.

[43] WU, L.; SHAHIDEHPOUR, M. ; LI, T.. Stochastic security-constrained unit commitment. IEEE Transactions on Power Systems, 22:800-811, 2007.

[44] TAKRITI, S.; BIRGE, J. R. ; LONG, E.. A stochastic model for the unit commitment problem. IEEE Transactions on Power Systems, 11, 1996. 
[45] SCHULZE, T.; GROTHEY, A. ; MCKINNON, K.. A stabilised scenario decomposition algorithm applied to stochastic unit commitment problems. European Journal of Operational Research, 261(1):247-259, 2017.

[46] PEREIRA, M. V. F.; PINTO, L. M. V. G.. Multi-stage stochastic optimization applied to energy planning. Mathematical Programming, 52:359-375, 1991.

[47] ZOU, J.; AHMED, S. ; SUN, X.. Stochastic dual dynamic integer programming. Mathematical Programming, 175:461-502, 2019.

[48] MODARRESI, M. S.; XIE, L.; CAMPI, M. C.; GARATTI, S.; CARE, A.; THATTE, A. A. ; KUMAR, P. R.. Scenario-Based Economic Dispatch with Tunable Risk Levels in High-Renewable Power Systems. IEEE Transactions on Power Systems, 34(6):5103-5114, 2019.

[49] MARGELLOS, K.; ROSTAMPOUR, V.; VRAKOPOULOU, M.; PRANDINI, M.; ANDERSSON, G. ; LYGEROS, J.. Stochastic unit commitment and reserve scheduling: A tractable formulation with probabilistic certificates. In: 2013 EUROPEAN CONTROL CONFERENCE (ECC), p. 2513-2518, 2013.

[50] VRAKOPOUlOU, M.; MARgELlOS, K. ; LYGEROS, J.. A Probabilistic Framework for Reserve Scheduling and N-1 Security Assessment of Systems With High Wind Power Penetration. IEEE Transactions on Power Systems, 28(4):1-12, 2013.

[51] FERnANDES, B.; STREeT, A.; VAlLADÃO, D. ; FERNANDES, C.. An adaptive robust portfolio optimization model with loss constraints based on data-driven polyhedral uncertainty sets. European Journal of Operational Research, 255(3):961-970, 2016.

[52] BERTSIMAS, D.; BROWN, D. B.. Constructing uncertainty sets for robust linear optimization. Operations Research, 57:1483-1495, 11 2009.

[53] BANDI, C.; BERTSIMAS, D.. Tractable stochastic analysis in high dimensions via robust optimization. Mathematical Programming, 134:23-70, 82012.

[54] BERTSIMAS, D.; SIM, M.. The price of robustness. Operations Research, 52(1):35-53, 2004. 
[55] GUAN, Y.; WANG, J.. Uncertainty sets for robust unit commitment. IEEE Transactions on Power Systems, 29(3):1439-1440, 2014.

[56] SUN, X.; LORCA, A.. Robust optimization in electric power systems operations. In: Du, P.; Baldick, R. ; Tuohy, A., editors, INTEGRATION OF LARGE-SCALE RENEWABLE ENERGY INTO BULK POWER SYSTEMS. Springer, 2017.

[57] PEREIRA, M. V. F.. Optimal stochastic operations scheduling of large hydroelectric systems. International Journal of Electrical Power \& Energy Systems, 11(3):161 - 169, 1989.

[58] OSTROWSKI, J.; ANJOS, M. F. ; VANNELLI, A.. Tight mixed integer linear programming formulations for the unit commitment problem. IEEE Transactions on Power Systems, 27(1):39-46, 2012.

[59] GEORGHIOU, A.; KUHN, D. ; WIESEMANN, W.. The decision rule approach to optimization under uncertainty: methodology and applications. Computational Management Science, 16:545-576, 2019.

[60] QIU, J.; DONG, Z. Y.; ZHAO, J.; XU, Y.; LUO, F. ; YANG, J.. A risk-based approach to multi-stage probabilistic transmission network planning. IEEE Transactions on Power Systems, 31(6):48674876, 2016.

[61] BODUR, M.; LUEDTKE, J. R.. Two-stage linear decision rules for multi-stage stochastic programming. Mathematical Programming, 2018.

[62] BIRGE, J. R.; LOUVEAUX, F.. Introduction to Stochastic Programming. Springer New York, New York, NY, 2011.

[63] ZHENG, Q.; WANG, J.; PARDALOS, P. ; GUAN, Y.. A decomposition approach to the two-stage stochastic unit commitment problem. Annals of Operations Research, 210:387-410, 2013. 